ARTICLE

https://doi.org/10.1038/s41467-019-13238-7

\title{
Zwitterionically modified alginates mitigate cellular overgrowth for cell encapsulation
}

Qingsheng Liu', Alan Chiu', Long-Hai Wang'1, Duo An (1) 1, Monica Zhong1, Alexandra M. Smink², Bart J. de Haan², Paul de Vos ${ }^{2}$, Kevin Keane (i) ${ }^{3}$, Andreas Vegge ${ }^{4}$, Esther Y. Chen ${ }^{5}$, Wei Song ${ }^{1}$, Wendy F. Liu ${ }^{5}$, James Flanders ${ }^{6}$, Claude Rescan7, Lars Groth Grunnet ${ }^{7}, X_{i}$ Wang $^{1} \&$ Minglin Ma (i] ${ }^{1 \star}$

Foreign body reaction (FBR) to implanted biomaterials and medical devices is common and can compromise the function of implants or cause complications. For example, in cell encapsulation, cellular overgrowth (CO) and fibrosis around the cellular constructs can reduce the mass transfer of oxygen, nutrients and metabolic wastes, undermining cell function and leading to transplant failure. Therefore, materials that mitigate FBR or CO will have broad applications in biomedicine. Here we report a group of zwitterionic, sulfobetaine (SB) and carboxybetaine (CB) modifications of alginates that reproducibly mitigate the $\mathrm{CO}$ of implanted alginate microcapsules in mice, dogs and pigs. Using the modified alginates (SBalginates), we also demonstrate improved outcome of islet encapsulation in a chemicallyinduced diabetic mouse model. These zwitterion-modified alginates may contribute to the development of cell encapsulation therapies for type 1 diabetes and other hormone-deficient diseases.

\footnotetext{
${ }^{1}$ Department of Biological and Environmental Engineering, Cornell University, Ithaca, NY 14853, USA. ${ }^{2}$ Department of Pathology and Medical Biology, University of Groningen and University Medical Center Groningen, Groningen, Netherlands. ${ }^{3}$ Stem Cell Biology, Novo Nordisk A/S, 2760 Måløv, Denmark. ${ }^{4}$ Diabetes Research, Novo Nordisk A/S, 2760 Måløv, Denmark. ${ }^{5}$ Department of Biomedical Engineering, University of California Irvine, Irvine, CA 92697, USA. ${ }^{6}$ Department of Clinical Sciences, Cornell University, Ithaca, NY 14853, USA. ${ }^{7}$ Stem Cell Pharmacology, Novo Nordisk A/S, 2760 Måløv, Denmark. *email: mm826@cornell.edu
} 
$\mathrm{T}$ ype 1 diabetes (T1D) affects millions of people worldwide, despite that many advanced therapeutic treatments have been developed ${ }^{1-8}$. To date, daily injection or infusion of exogenous insulin is still the leading treatment option to provide blood glucose (BG) control for people with T1D ${ }^{9}$. However, insulin therapies are tedious, often associated with patient compliance and cannot totally prevent diabetic side effects ${ }^{10}$. Pancreatic islet transplantation has worked for some patients ${ }^{11}$, but it is limited to only a small fraction of patients because of a shortage of donor islets and the need for long-term immuno-suppression. Recently, human stem cell-derived beta (SC- $\beta$ ) cells have been developed, providing a pathway to produce an unlimited supply of insulin-producing cells ${ }^{12,13}$. However, these cells still need to be immunoprotected or encapsulated to prevent the immune and autoimmune responses.

Cell encapsulation has indeed shown great promise in numerous animal studies. Among the different materials used for cell encapsulation, alginate is one of the most prevalent ones to date ${ }^{14-16}$, due in a large part to its mild gelation conditions and minimal toxicity ${ }^{15-18}$. However, foreign body reaction (FBR), a complex process involving protein adsorption, monocyte/granulocytes/macrophage adhesion, giant cell formation, and crosstalks between macrophages/giant cells and other immune/fibroblast cells, against alginate microcapsules is often observed and can be further elevated by encapsulated cells or xenogeneic donor tissue ${ }^{5,19,20}$. The cellular overgrowth (CO) and the fibrosis, an end result of the $\mathrm{FBR}^{21,22}$ that the body forms to isolate foreign implants reduce and even cut off the diffusion of nutrients and oxygen to the encapsulated cells, causing cell necrosis. To mitigate the $\mathrm{CO}$ of alginate microcapsules, investigators recently took an expensive, time-consuming but effective high throughput approach. Vegas et al. created a library of almost 800 chemically modified alginate derivatives and identified a few "hits" (e.g., Z1Y15 containing triazole group) that effectively mitigated $\mathrm{CO}$ in mice and non-human primates 23,24 .

We report here a totally different, more rational and much less expensive approach to develop CO-mitigating, chemically modified alginates. Nonspecific protein adsorption onto implanted material is considered the first and critical step of $\mathrm{FBR}^{25-27}$. An antifouling material or surface that is highly resistant to protein adsorption and cell attachment is expected to suppress FBR and subsequently the $\mathrm{CO}$ and formation of fibrosis ${ }^{25}$. Recently, zwitterionic polymers, bearing zwitterions of carboxybetaine (CB), sulfobetaine (SB) and phosphorycholine, have been extensively studied in regards to their ultra-low-fouling properties ${ }^{28-30}$. For example, zwitterionic poly(carboxybetaine methacrylate) (PCBMA) hydrogels have been shown to resist the formation of fibrotic capsule for at least 3 months after subcutaneous implantation in mice ${ }^{26}$. Based on these previous studies, we rationalized that chemically modifying alginate with zwitterionic groups might lead to a different class of $\mathrm{CO}$-mitigating alginate derivatives.

We first modify alginates (Ultrapure VLVG, SLG20, SLG100) with a zwitterionic group, SB and find that the modification reproducibly reduces the $\mathrm{CO}$ of the alginate microcapsules (diameter: $500 \sim 700 \mu \mathrm{m}$ ) in different species: C57BL/6J mice (intraperitoneal implantation), dogs (intraperitoneal) and pigs (omental pouch). To show the observed effect is reproducible, we have done a total of 17 mouse experiments with different types of alginates and different time points up to 6 months. Consistently, the SB-alginate microcapsules induce significantly less CO than the unmodified control and most of the times almost free of CO. Interestingly, the CO-mitigating effect of the zwitterionic modification is also observed in carboxybetaine-based alginates (i.e., CB-alginates). Additional experiments in large animals including dogs and pigs show similarly reduced $\mathrm{CO}$ of the SB-alginate compared to the unmodified SLG20 or SLG100, indicating the potential translatability of the zwitterionic modification. Then we encapsulate rat islets using either the SB-alginate microcapsules or unmodified control microcapsules and transplant them intraperitoneally in C57BL/6J mice with streptozotocin (STZ)induced diabetes. The SB-alginate microcapsules result in significantly better long-term glycemic control, up to 200 days. Characterization of retrieved microcapsules and islets confirms the CO-mitigating property of the SB-alginate microcapsules as well as islet survival and function. Compared with the previously published high throughput approach, the zwitterionic modification represents a much simpler and less expensive strategy for the design and development of super-biocompatible alginates. We believe that these zwitterionically modified-alginates and our approach may contribute to a cell encapsulation therapy for T1D and potentially other hormone-deficient diseases in the future.

\section{Results}

Development of zwitterionically modified alginates. Recently, zwitterionic polymers and hydrogels have been extensively investigated due to their attractive ultra-low biofouling and biocompatible characteristics ${ }^{28}$. However, harsh required conditions such as UV irradiation or generation of free radical groups during the gelation of zwitterionic materials can be harmful to encapsulated cells, limiting broad biomedical applications ${ }^{31-33}$. We hypothesized that we could overcome this limitation but maintain the biocompatibility of zwitterionic compounds by developing a group of zwitterionically modified alginates. In these alginate derivatives, the zwitterionic moiety provides high surface hydration $^{34}$, resistance to protein adsorption or cell adhesion, and mitigation of $\mathrm{CO}$, while the alginate backbone remains crosslinkable with mild gelation condition and allows formation of microcapsules using an electrospraying technique (Fig. 1a).

Among zwitterionic groups, the SB group was firstly chosen in our studies because of its excellent antifouling performance, commercial availability and low $\operatorname{cost}^{35,36}$. In order to modify the alginate with SB group, we designed and synthesized SB- $\mathrm{NH}_{2}$ monomer according to Supplementary Fig. 1. Then, we chose low molecular weight (MW), ultrapure alginate VLVG as the starting material. 2-chloro-4, 6-dimethoxy-1, 3, 5-triazine (CDMT) and $\mathrm{N}$-methylmorpholine (NMM) were used as coupling reagents to conjugate alginate with $\mathrm{SB}-\mathrm{NH}_{2}$ via a triazine-based coupling reaction (Fig. 1b). The SB-based alginate conjugate was characterized by ${ }^{1} \mathrm{H}$ NMR spectrum (Fig. 1b) where a peak at $3.20 \mathrm{ppm}$ was attributed to the six protons in two methyl groups attached to the quaternary amine in the SB pendant group. The result suggested that $\mathrm{SB}-\mathrm{NH}_{2}$ was successfully conjugated to alginate. About 30.5\% modification degree of the starting alginate was confirmed by NMR data analysis. Using similar procedures, we also modified higher molecular weight alginates, SLG20 and SLG100.

To examine how the zwitterionic modifications may have affected the physiochemical properties of the alginates and microcapsules, we performed a number of characterizations. First, the surface roughness of SB-SLG20 and SLG20 microcapsules, assessed by atomic force microscope (AFM), were $11 \pm 1 \mathrm{~nm}$ and $17 \pm 15 \mathrm{~nm}$, respectively (Supplementary Fig. 2). The SB-SLG20 capsules appeared slightly smoother but these two kinds of alginate capsules had no statistical difference in the surface roughness. We then evaluated whether this zwitterion modification changed the surface charge. Zeta-potentials of SLG20 and SB-SLG20 hydrogels were $-17.3 \pm 0.5$ and $-12.2 \pm 0.3 \mathrm{mV}$, respectively (Supplementary Fig. 3). Zeta-potentials of SB-SLG20 and SLG20 hydrogels were similar and they are both negatively charged polymers. To compare the mass transfer of the unmodified and modified alginate 

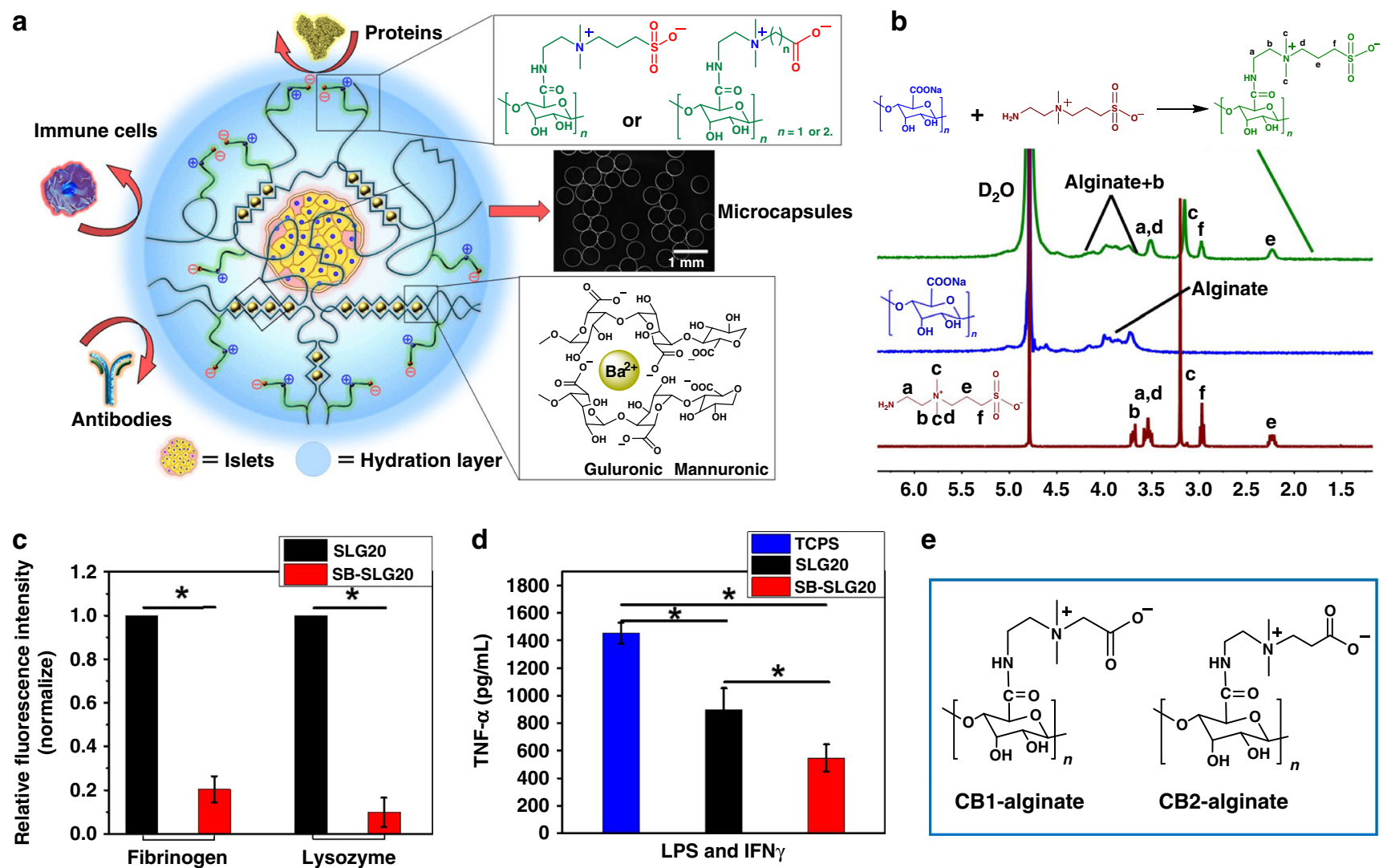

Fig. 1 Design of zwitterionically modified alginates and their in vitro characterizations. a Schematic illustration of zwitterionically modified alginate microcapsules encapsulating islets. b Synthetic pathway and ${ }^{1} \mathrm{H}$ NMR characterization of sulfobetaine (SB)-modification of alginate. c Adsorption of FITClabeled fibrinogen and lysozyme on the surfaces of different alginate hydrogels quantified by ImageJ. Mean \pm SEM; $n=6 ;{ }^{\star} P<0.05$. $\mathbf{d}$ Quantification of TNF- $\alpha$ secretion from macrophages cultured on different surfaces. Mean $\pm S E M ; n=5 ;{ }^{\star} P<0.05$. e Chemical structures of $C B 1$-alginate and $C B 2$-alginate conjugates

hydrogels, we immersed SLG20 and SB-SLG20 hydrogels into different molecular weight, FITC-labeled dextran standards, respectively. The results (Supplementary Fig. 4) indicate that the diffusion rate of SB-SLG20 hydrogel was similar to that of SLG20 hydrogel regardless of molecular weight of dextrans. Since the mechanical property of the microcapsules is an important consideration in the success of cell encapsulation, it was evaluated in our studies by a Texture Analyzer. SB-SLG20 microcapsules under force were slightly stronger than SLG20 microcapsules (Supplementary Fig. 5). This might be attributed to the $40 \%$ SLG100 alginate (which has a larger molecular weight than SLG20) addition during preparation of the SB-SLG20 solution. Taken together, the zwitterionic modification did not seem to change the physiochemical properties of the microcapsules significantly.

Protein adsorption on the surface of an implanted medical device is the first step in a foreign body response, which will eventually affect the performances of the device ${ }^{25,37}$. Therefore, protein adsorption on the modified alginate was studied in our work, with unmodified SLG20 alginate as control. Two model proteins, fibrinogen $(340 \mathrm{kDa}$, isoelectronic point: 5.5$)$ and lysozyme ( $14 \mathrm{kDa}$, isoelectronic point: 11.1$)$, were used to study the adsorption on the alginate hydrogel surfaces. These model proteins represent different molecular weights, structural stability, and isoelectronic points. Relative to SLG20 hydrogels, the amount of fibrinogen and lysozyme adsorptions on SB-SLG20 is 20.3 and 9.8\%, respectively (Fig. 1c and Supplementary Fig. 6), indicating a strong resistance to non-specific protein adsorption. The excellent antifouling property of SB-SLG20 is probably due to the strong hydration of the SB groups ${ }^{34}$.
We then studied macrophage activation on the modified alginate hydrogels by seeding murine bone marrow derived macrophages (BMDM) and examining release of tumor necrosis factor- $\alpha$ (TNF- $\alpha)$ as a representative pro-inflammatory cytokine. After stimulation with lipopolysaccharide/interferon gamma (LPS/IFN $\gamma$ ) which is known to induce a proinflammatory macrophage phenotype ${ }^{38}$, the BMDMs cultured on the SB-SLG20 hydrogels secreted lower levels of TNF- $\alpha$ when compared to those cultured on the SLG20 hydrogels or tissue culture polystyrene plates (TCPS) (Fig. 1d). This study demonstrated that incorporating a zwitterionic moiety into alginate effectively inhibited the inflammatory activation of macrophages in vitro.

We also studied the impact of the alginate microcapsules on tolllike receptors (TLRs) signaling. TLRs are a class of proteins that play a key role in the innate immune system ${ }^{39}$. We used human embryonic kidney (HEK) cell line that expresses specific TLR signaling. The SLG20 and SB-SLG20 microcapsules (Supplementary Fig. 7) did not activate TLR2 or TLR4 but they did inhibit the signaling, indicating SLG20 and SB-SLG20 hydrogels were not immunostimulatory. More interestingly, SB-SLG20 capsules were shown to inhibit TLR2, more than SLG20 capsules and the control. These results again point to the potential anti-inflammatory effect of the zwitterionic modification.

To explore whether the in vitro anti-fouling and antiinflammatory properties translate into $\mathrm{CO}$ mitigation in vivo, we performed a number of animal experiments. In addition to the SB-alginate, we also designed, synthesized and tested two other kinds of zwitterionically modified alginates (CB1-alginate and 


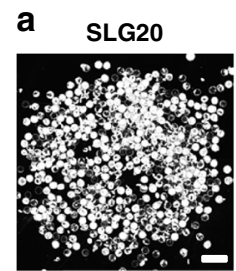

SLG20

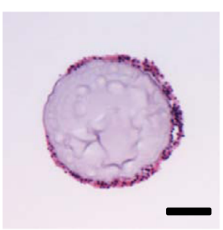

b

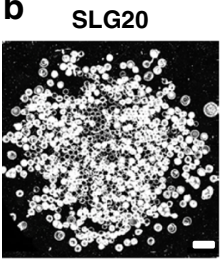

SLG20

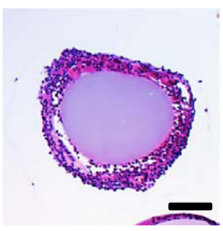

SB-VLVG

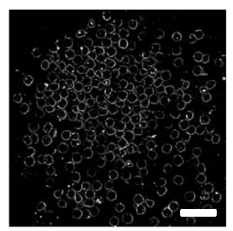

SB-VLVG

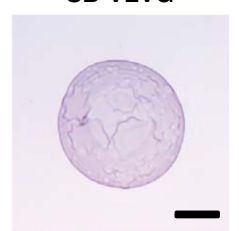

SB-SLG20

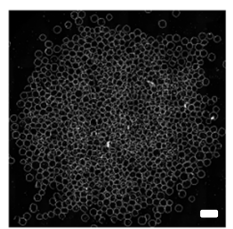

SB-SLG20

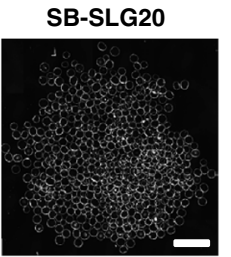

SB-SLG20
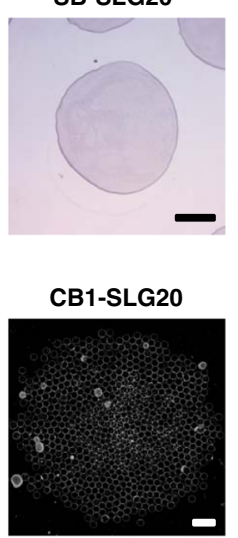

CB1-SLG20

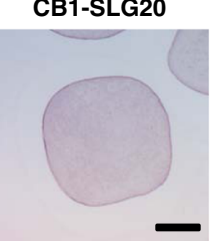

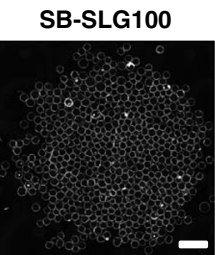

SB-SLG100

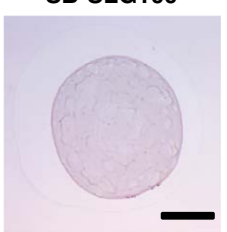

CB2-SLG2O

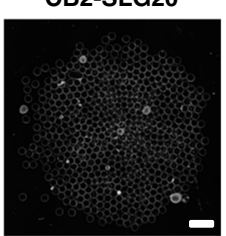

CB2-SLG2O

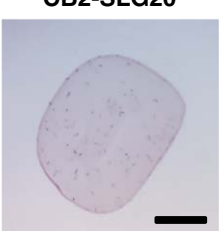

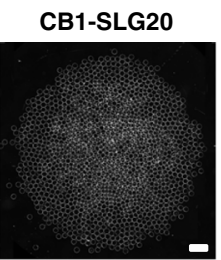

CB1-SLG20
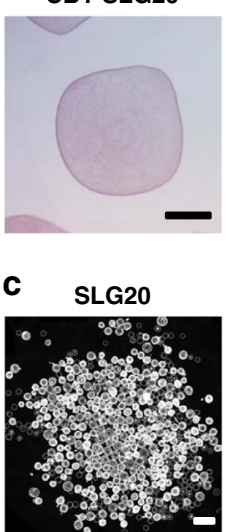

SLG20

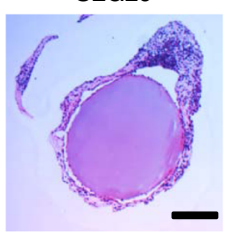

CB2-SLG20

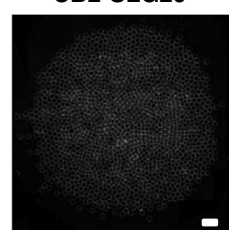

CB2-SLG20

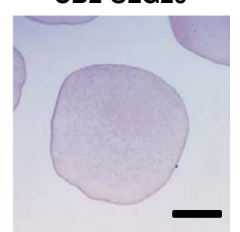

SB-SLG20

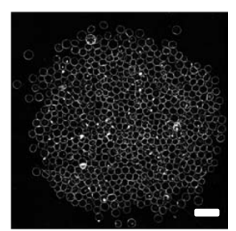

SB-SLG20

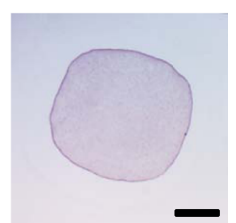

Fig. $2 \mathrm{SB}$ and $\mathrm{CB}$ modified alginates mitigate $\mathrm{CO}$ in mice. a Representative phase-contrast images of retrieved microcapsules made from different alginates ( $S L G 20, n=19$, see Supplementary Fig. 11 for complete data; SB-VLVG, $n=10$, see Supplementary Fig. 12 for complete data; SB-SLG20, $n=16$, see Supplementary Fig. 14 for complete data; SB-SLG100, $n=10$, see Supplementary Fig. 15 for complete data; CB1-SLG20, $n=5$, see Supplementary Fig. 16a for complete data; CB2-SLG20, $n=5$, see Supplementary Fig. 16b for complete data), 14 d post intraperitoneal implantation in C57BL/6J mice (Scale bar, $2000 \mu \mathrm{m}$ ) and corresponding H\&E stained histological analysis (Scale bar, $200 \mu \mathrm{m}$ ). b Representative phase-contrast images of retrieved microcapsules, $100 \mathrm{~d}$ post intraperitoneal implantation in C57BL/6J mice (Scale bar, $2000 \mu \mathrm{m}$ ) and corresponding H\&E stained histological analysis (Scale bar, $200 \mu \mathrm{m}$ ). (SLG20, $n=12$, see Supplementary Fig. 17 for complete data; SB-SLG20, $n=10$, see Supplementary Fig. 18 for complete data; CB1-SLG20, $n=4$, see Supplementary Fig. Supplementary Fig. 20a for complete data; CB2-SLG2O, $n=4$, see Supplementary Fig. 20b for complete data). c Representative phasecontrast images of retrieved microcapsules, $180 \mathrm{~d}$ post intraperitoneal implantation in C57BL/6J mice (Scale bar, $2000 \mu \mathrm{m}$ ) and corresponding H\&E stained histological analysis (Scale bar, $200 \mu \mathrm{m}$ ). (SLG20, $n=7$, see Supplementary Fig. 22 for complete data; SB-SLG20, $n=7$, Supplementary Fig. 21 for complete data)

CB2-alginate, Fig. 1e; see Supplementary Figs. 8 and 9 for related $\mathrm{NH}_{2}$ terminated monomers $\mathrm{CB} 1-\mathrm{NH}_{2}$ and $\mathrm{CB} 2-\mathrm{NH}_{2}$ ).

Zwitterionically modified alginates mitigate $\mathrm{CO}$ in mice. To evaluate the biocompatibility of SB-modified alginates, we first chose immunocompetent C57BL/6J mice because this strain was previously shown to elicit a strong $\mathrm{CO}$ against unmodified alginate microcapsules ${ }^{40}$. Microcapsules of SB-VLVG alginate were fabricated using electrospraying technique and had a uniform spherical morphology and diameters ranging from 450 to $550 \mu \mathrm{m}$, as shown in Fig. 1a and Supplementary Fig. 10. Unmodified SLG20 alginate was processed into microcapsules with similar size and morphology and used as control. The microcapsules were implanted in the intraperitoneal space of C57BL/6J mice and retrieved for characterization 14 days post implantation.

To characterize the $\mathrm{CO}$, we fused dark-field microscopic images of all retrieved microcapsules to obtain a composite view. In the images, the whiteness on the microcapsule surfaces (Fig. 2a) indicated the cellular deposition. Clearly, the control microcapsule (SLG20) as shown in Fig. 2a induced variable and substantial cellular deposition (see Supplementary Fig. 11 for all other 18 samples from 6 batches with a total $n=19$ ), which was consistent with that of previously reported work ${ }^{41}$. In contrast, there was almost no cellular deposition observed on the SBVLVG alginate microcapsules (Fig. 2a and see Supplementary Fig. 12 for all other 9 samples from three batches with a total $n=$ $10)$. The representative $H \& E$ staining of microcapsule crosssections (Fig. 2a) further confirmed that the surfaces of SB-VLVG alginate microcapsules were almost free of $\mathrm{CO}$ while the surfaces of SLG20 microcapsules had visible CO. To examine whether the observation was reproducible, another batch of SB-VLVG alginate was synthesized and evaluated for CO. This batch of retrieved SB-VLVG alginate as shown in Supplementary Fig. 12 also exhibited almost no cellular deposition. These results suggest that SB-VLVG alginate microcapsules mitigated CO effectively and reproducibly. This may be attributed to the zwitterionic SB group that increases surface hydration, reduces biofouling and improves biocompatibility of alginate. We have also compared directly one of the "hits" from the library screening 23 with SB-VLVG alginates. As shown in Supplementary Fig. 13, there were minimal and similar levels of cellular depositions on our modified alginate and the one based on previous library screening method $^{23}$, suggesting comparable biocompatibility despite different chemistries and approaches. 
a

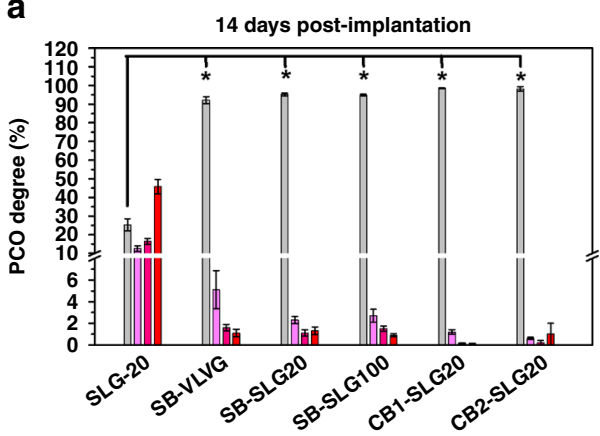

b

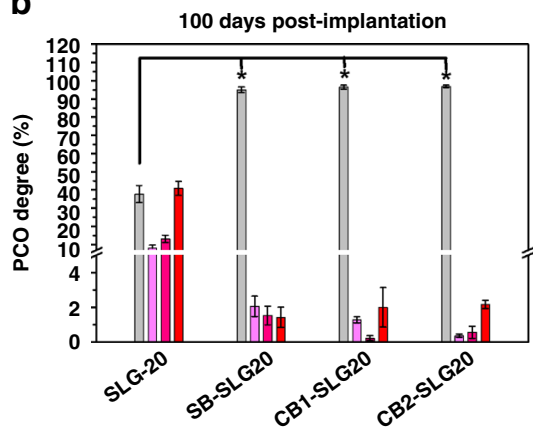

C

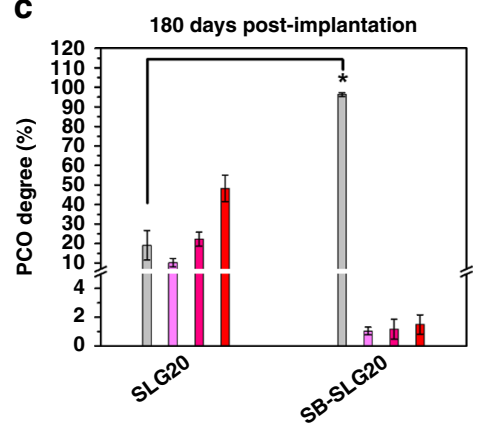

$0-25 \%$ capsule PCO

$25-50 \%$ capsule PCO

$50-75 \%$ capsule PCO

$75-100 \%$ capsule PCO

Fig. 3 PCO evaluation of retrieved microcapsules. a Quantification of PCO for retrieved microcapsules, $14 \mathrm{~d}$ post implantation. Mean $\pm \mathrm{SEM}$; $n=19$ for SLG20; $n=10$ for SB-VLVG; $n=16$ for SB-SLG20; $n=10$ for SB-SLG100; $n=5$ for CB1-SLG20; $n=5$ for CB2-SLG20. ${ }^{\star} P<0.05$. b Quantification of PCO for retrieved microcapsules, $100 \mathrm{~d}$ post implantation. Mean $\pm \mathrm{SEM} ; n=12$ for SLG20; $n=10$ for SB-SLG20; $n=4$ for CB1-SLG20; $n=4$ for CB2-SLG20. ${ }^{\star} P<$ 0.05. c Quantification of PCO for retrieved microcapsules, $180 \mathrm{~d}$ post implantation. Mean \pm SEM; $n=7$ for SLG20; $n=7$ for SB-SLG20. ${ }^{\star}<<0.05$

To verify that the CO-mitigating effect of SB modification does not depend on the type of alginate, we also synthesized SB-SLG20 alginate and SB-SLG100 alginate using the same method. Due to their higher molecular weights, the SLG20 (MW: 75-150 kDa) and SLG100 (MW: 150-250 kDa) form stronger hydrogels than VLVG alginate $(\mathrm{MW}<75 \mathrm{kDa})$, and may have broader applications in biomedicine. The SB-SLG20 and SB-SLG100 alginate microcapsules were implanted in the intraperitoneal space of C57BL/6J mice and then retrieved after 14 days, with SLG20 microcapsules as control. Dark-field images of all retrieved SBSLG20 alginate microcapsules $(n=16$; Fig. $2 \mathrm{a}$ and all other samples from three batches in Supplementary Fig. 14) and SBSLG100 microcapsules ( $n=10$; Fig. 2a and all other samples from two batches in Supplementary Fig. 15) showed very little CO. $\mathrm{H} \& \mathrm{E}$ staining (Fig. 2a) also indicated that there was almost no CO around retrieved SB-SLG20 and SB-SLG100 alginate microcapsules. In contrast, the conventional, unmodified SLG20 microcapsules induced varied degrees of $\mathrm{CO}$, and some elicited severe CO. To explore whether other zwitterionic moieties play a similarly important role in mitigating $\mathrm{CO}$, the biocompatibility of CB-alginates was evaluated. Interestingly, CB1-SLG20 and CB2SLG20 (Fig. 2a and see Supplementary Fig. 16 for all other samples; $n=5$ from one batch) microcapsules were also found to have little or no CO. Taken together, these results suggest that the $\mathrm{CO}$-mitigation of zwitterionic modification is reproducible, and independent of alginate or zwitterion types, at least during 2-week intraperitoneal implantation in mice.

To investigate whether the modification can mitigate $\mathrm{CO}$ for a much longer term, we implanted SB-SLG 20 and CB-SLG20 microcapsules in $\mathrm{C} 57 \mathrm{BL} / 6 \mathrm{~J}$ mice and retrieved them after 100 days. Retrieved SLG20 microcapsules (Fig. 2b and Supplementary Fig. 17, $n=12$ from three batches) exhibited significant cellular deposition, which was further verified by histological analysis (Fig. 2b). Moreover, some of the retrieved SLG20 microcapsules even aggregated together, a sign of severe FBR (Supplementary Fig. 17). However, SB-SLG20 microcapsules (Fig. $2 \mathrm{~b}$ and Supplementary Fig. 18, $n=10$ from two batches) had a much lower level of cellular deposition, consistent with histology results (Fig. 2b). We also verified that SB-SLG100 microcapsules mitigated the $\mathrm{CO}$ effectively at 100 days (Supplementary Fig. 19, $n=4$ ) indicating that this CO-mitigating zwitterionic modification is independent of alginate types for long-term implantation. Moreover, CB1-SLG20 and CB2-SLG20 microcapsules (Fig. 2b and Supplementary Fig. 20, $n=4$ ) also had almost no CO. To further evaluate the longevity of the CO- resistant property, SB-SLG20 microcapsules were examined 180 days after implantation in $\mathrm{C} 57 \mathrm{BL} / 6 \mathrm{~J}$ mice. As shown in Fig. 2c and Supplementary Fig. $21(n=7)$, SB-SLG20 microcapsules were largely free of cellular deposition after retrieval, which was consistent with H\&E staining. However, there was severe CO observed on the surfaces of unmodified SLG20 microcapsules (Fig. 2c and Supplementary Fig. 22, $n=7$ ). A striking difference between the modified and unmodified microcapsules was also observed in macroscopic photos of the retrieved samples (Supplementary Fig. 21b and 22b). The retrieved SLG20 microcapsules appeared mostly white in Petri dishes, indicating severe cellular deposition (Supplementary Fig. 22b, bottom two rows), while the near transparent appearance of the retrieved SB-SLG20 microcapsules suggested negligible CO (Supplementary Fig. 21b, bottom two rows).

To quantify the observations described above, we calculated retrieval rates (Supplementary Table 1) and categorized all retrieved microcapsules based on the percentage of surface coverage by "pericapsular cellular overgrowth" or $\mathrm{PCO}^{24,42,43}$ : $0-25,25-50,50-75$, and $75-100 \%$. For SLG20 control microcapsules after 14 days implantation (Fig. 3a), the $0-25 \%$ PCO category (a sign of no or little CO) accounted for $24.5 \%$ of all retrieved microcapsules, and the $75-100 \%$ PCO category (a sign of severe CO) made up to $42.5 \%$. In contrast, more than $90 \%$ of all retrieved zwitterionically modified microcapsules fell into the 0-25\% PCO category, a significant improvement over conventional SLG20 microcapsules. Similarly, during long-term (100-180 days) studies (Figs. 3b, c), 90\% of modified alginate microcapsules developed minimal CO (i.e. within the $0-25 \%$ PCO category), while almost half of SLG20 control microcapsules had severe CO (i.e., within the $75-100 \%$ PCO category). These quantifications suggest that zwitterionic SB and CB modifications substantially reduced $\mathrm{CO}$ of alginate microcapsules in the intraperitoneal space of C57BL/6J mice in both short $(14 \mathrm{~d})$ and long terms (100 d). More remarkably, zwitterionic SB-SLG20 was shown to mitigate the $\mathrm{CO}$ effectively, up to half a year.

Lastly, to better understand the phenotypes of adhered cells on the retrieved microcapsules and the innate immune response caused by zwitterion-modified and unmodified alginates, we implanted SLG20 and SB-SLG20 microcapsules in C57BL/6 mice for 2 weeks and immunologically analyzed the capsules and the intraperitoneal fluid surrounding the capsules. The retrieved capsules were stained by a number of cellular markers including a-smooth muscle actin (SMA), CD68, F4/80, CD11b, and Ly-6G/ Ly-6C (Supplementary Figs. 23-26). These staining experiments 
revealed that the cells attached to microcapsules included monocytes, granulocytes, macrophages and fibroblasts, and there was a significant reduction of adhesion of these cells, particularly monocytes and neutrophils, after the zwitterionic modification consistent with the phase-contract images.

From immune profiling of the peritoneal fluid 2 weeks post implantation with 40 different cytokines (Supplementary Fig. 27), we observed interestingly that samples fabricated with modified alginate contained less inflammatory cytokines/components/chemokines in the intraperitoneal fluid than those with unmodified control, including C5/C5a, IP-10, TREM-1, IL-1 $\beta$, IL-1a, CCL1, CCL2, CCL $3{ }^{44,45}$. TIMP-1 was also downregulated in the samples made with modified alginate, which inhibits matrix metalloproteinase and promotes fibrosis ${ }^{46}$. Another interesting observation was that the unmodified alginate samples contained CXCL1, CXCL2, CXCL12, which are powerful neutrophil chemoattractants that are involved in many immune responses including wound healing, cancer metastasis, and angiogenesis ${ }^{47}$. The results from immunostaining also verified the neutrophil trafficking in unmodified samples (Supplementary Fig. 26). Both modified and unmodified samples contained similar levels of chemokine CXCL13, which attracts $\mathrm{B}$ cells in peritoneum and promotes antibody production ${ }^{48}$. M-CSF (CSF1), secreted by macrophages and fibroblasts, is similarly expressed in both samples and important for the survival and proliferation of macrophages, confirming the local milieu of fibroblasts and macrophages ${ }^{49}$. IL16, also expressed by both samples, is a lymphocyte chemoattractant factor for $\mathrm{CD}^{+}$lymphocytes, which not only regulates migration of all $\mathrm{CD}^{+} \mathrm{T}$ cells but also facilitates the expansion of $\mathrm{CD}^{+} \mathrm{CD} 25^{+}$Treg cells ${ }^{50}$. In summary, the immune profiling results seem to suggest that the zwitterionic modification influenced the cytokine expression in the intraperitoneal fluids surrounding the capsules and downregulated several inflammatory cytokines particularly neutrophil chemoattractants. More work will be needed in the future to fully understand the exact roles of all the cytokines we profiled in the host responses against the microcapsules.

FBR mitigation in dogs and pigs. Next, we explored whether the observations in mice would translate to large animals such as dogs and pigs. First, SB-SLG20 microcapsules $(\sim 500 \mu \mathrm{m})$ were implanted intraperitoneally into Beagle dogs $(n=3)$ using a minimally invasive laparoscopic procedure. Efforts were made to spread out the microcapsules as much as possible. Unmodified SLG20 microcapsules were also implanted in one dog as control. The biocompatibility of the microcapsules was assessed 45 days after implantation using a similar laparoscopic procedure. There was no visible adhesion of SB-SLG20 microcapsules to host tissue (Fig. 4a), and they were easily dissociated from the implant site using either saline washing or catheter manipulation. A fraction of the microcapsules were aspirated out for characterization (Supplementary Movie 1). In contrast, the SLG20 microcapsules mostly adhered to the surrounding tissues and some were even fully embedded (Fig. 4b), making retrieval difficult. Multiple aspirations were needed to retrieve a sufficient number of microcapsules for characterization (Supplementary Movie 2). Dark-field microscopic images of retrieved SB-SLG20 microcapsules (Fig. 4c and Supplementary Fig. 28) revealed negligible cellular deposition, which was evidenced by the near transparent macroscopic appearance (Fig. 4d and Supplementary Fig. 28b). $\mathrm{H} \& \mathrm{E}$ stained section of retrieved capsules confirmed that there was minimal cellular deposition on the surfaces (Fig. 4e). In the contrast, the retrieved SLG20 microcapsules showed presence of strong CO (Fig. 4f) and many of them were covered with multiple cell layers (Fig. 4g). Moreover, we assessed the SB-SLG20 microcapsules again at 90 days post implantation from 2 of the 3 dogs that received implants. Still, the microcapsules had almost no tissue adhesion (Fig. 4h) and were mostly free of cellular deposition (Fig. 4i, j, and Supplementary Fig. 29).

To further evaluate the FBR to SB-alginate microcapsules, we chose insulin treated type 1 streptozotocin (STZ)-induced Göttingen minipigs and implanted SB-SLG100 microcapsules (Size: $500 \sim 700 \mu \mathrm{m})$ into pig omental bursa $(n=2)$, which is known to be extremely prone to elicit FBR following surgical intervention (Supplementary Fig. 30). In contrast to the laparoscopic implantation in dogs, the microcapsules were implanted as a whole without being spread out inside the omentum opening. (See Supplementary Fig. 30 for surgical details.) Unmodified SLG100 microcapsules were implanted as control $(n=2)$. One month after implantation, we excised the omentum and histologically analysed the microcapsules that were embedded. While both types of microcapsules caused FBR (as expected in such a fibrotic environment), there appeared to be differences. Unmodified SLG100 microcapsules had a dense and thick collagen deposition as indicated by the dark blue color with Masson's trichrome staining and also induced a great number of inflammatory cells as indicated by the red color (Fig. $4 \mathrm{k}$ and Supplementary Fig. 31a for two pigs, respectively). On the contrary, SB-SLG100 microcapsules were observed to have a looser and thinner collagen deposition and were covered with a smaller number of inflammatory cells, as shown in Fig. 41 and Supplementary Fig. 31b. Moreover, periodic acid-schiff (PAS) staining of retrieved tissue showed that unmodified SLG100 microcapsules (Fig. $4 \mathrm{~m}$ and Supplementary Fig. 32a) were generally associated with thicker and more mature bands of fibrous connective tissue, and had a marked FBR which included a chronic-active inflammatory cell infiltrate (lymphocytes and neutrophils), reactive fibroplasia, and foreign body giant cells (Fig. 4m, arrow). SB-SLG100 microcapsules (Fig. $4 \mathrm{n}$ and Supplementary Fig. 32b) had thinner and more wispy bands of connective tissue, and had a relatively reduced FBR including reduced fibroplasia, fewer chronic inflammatory cells (lymphocytes) and fewer/smaller multinucleated cells. H\&E staining of cross-sections (Supplementary Fig. 33) confirmed that there was less cellular infiltration among the SB-SLG100 microcapsules compared with the SLG100 control. While more experiments with a larger $n$ are required to perform quantitative, statistical comparisons, qualitatively the SB-alginate was shown to induce less FBR than the control even in a challenging, pro-fibrotic environment. All the results from mice, dogs, and pigs combined together point to the FBR or CO-mitigation effect of zwitterionic modifications for alginate microcapsules across species and at different implantation sites.

Improvement of diabetes treatments in mice. After confirming that the zwitterionically modified alginates SB-SLG20 mitigated FBR in C57BL/6J mice and large animals, we explored its therapeutic potential as a cell encapsulation medium for treatment of T1D. SB-SLG20 microcapsules (Size: $800 \sim 1000 \mu \mathrm{m}$; the size distribution as shown in Supplementary Fig. 34) encapsulating rat islets (500 islet equivalents per mouse) were transplanted into the peritoneal cavity of streptozotocin (STZ)-induced C57BL/6J diabetic mice and evaluated for 90 days for their ability to restore normoglycemia. Rat islets were also encapsulated in unmodified SLG20 microcapsules as control (Fig. 5a). The BG level of the mice decreased to normal glycemic range $(B G<200 \mathrm{mg} / \mathrm{dL})$ a few days after transplantation (Fig. 5b) for both groups. However, mice from the control group (i.e., unmodified microcapsules) experienced a shorter duration of glycemic control and four out of the six mice were unable to sustain normoglycemia within 
a

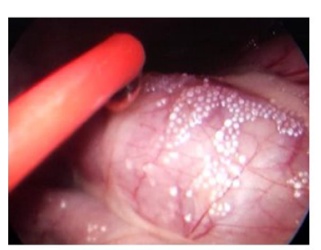

b

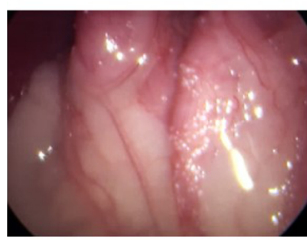

h

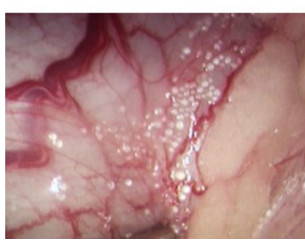

C

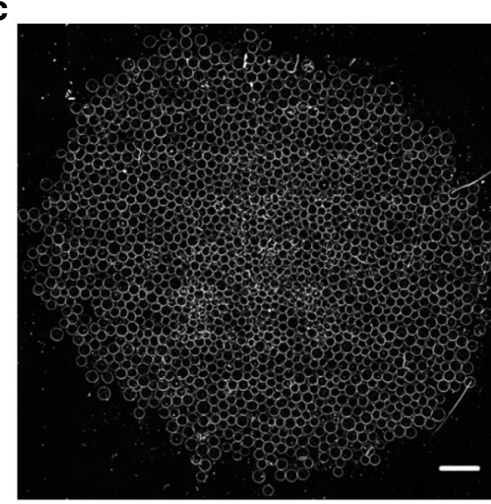

d

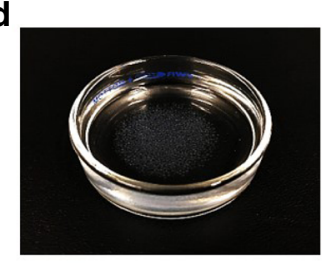

e

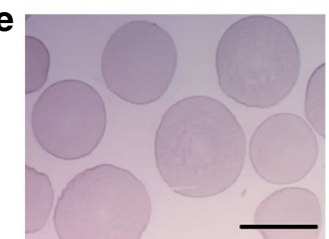

f

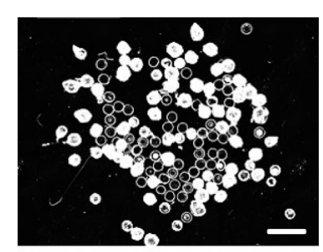

g

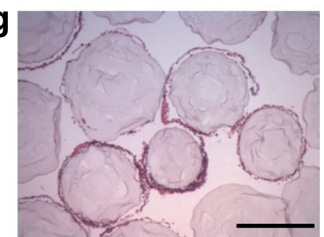

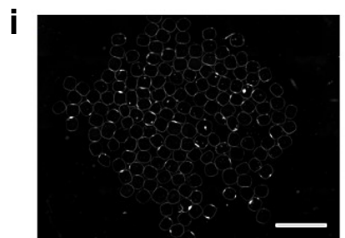

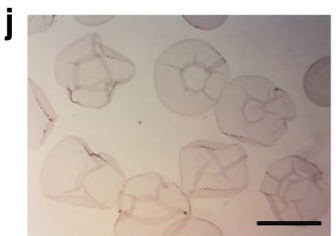

k

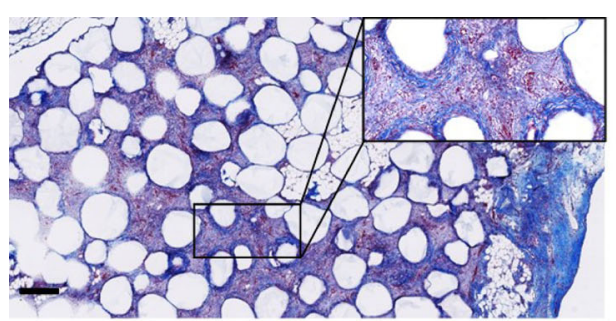

m

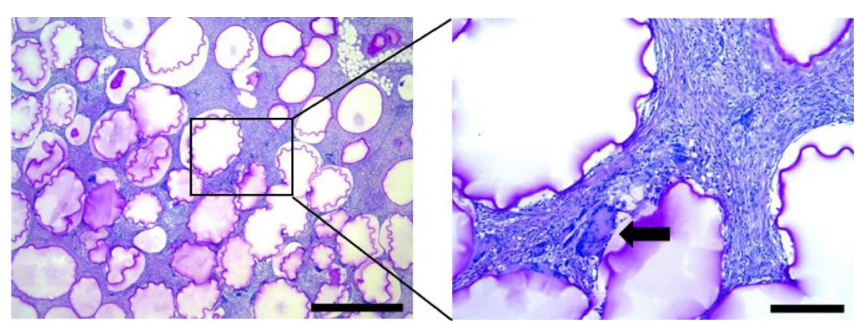

I

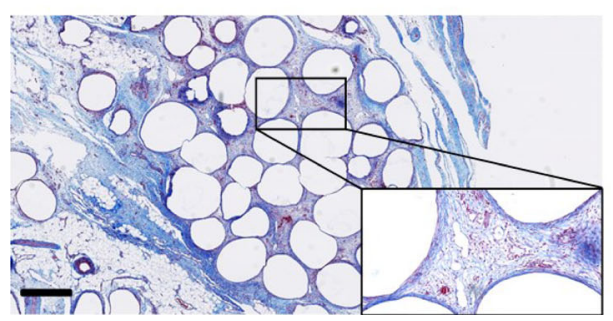

n

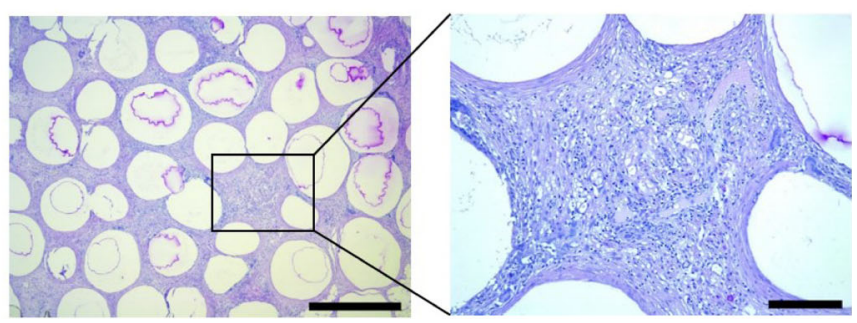

Fig. 4 SB modified alginates mitigate FBR in dogs and pigs. a A laparoscopic image during retrieval of SB-SLG20 alginate microcapsules, 45 days after intraperitoneal implantation in a dog. b A laparoscopic image during retrieval of SLG20 control microcapsules. c A phase contrast image of retrieved SBSLG20 microcapsules ( $n=3$; scale bar, 2 mm; see Supplementary Fig. 28 for complete data). d Retrieved SB-SLG20 microcapsules in a Petri dish. e H\&E stained cross-sectional image of retrieved SB-SLG20 microcapsules (Scale bar, $500 \mu \mathrm{m})$. f A phase contrast image of retrieved SLG20 microcapsules $(n=$ 1; scale bar, $2 \mathrm{~mm}$ ). $\mathbf{g} \mathrm{H \& E}$ stained cross-sectional image of retrieved SLG20 microcapsules (Scale bar, $500 \mu \mathrm{m}$ ). $\mathbf{h}$ A laparoscopic image during retrieval of SB-SLG20 alginate microcapsules, 90 days after intraperitoneal implantation in a dog. i A phase contrast image of retrieved SB-SLG20 microcapsules ( $n=$ 2; scale bar, 2 mm; see Supplementary Fig. 29 for complete data). j H\&E stained cross-sectional image of retrieved SB-SLG20 microcapsules (Scale bar, $500 \mu \mathrm{m}$ ). k Representative Masson's trichrome staining (and a higher magnification) images of retrieved SLG100 alginate microcapsules ( $n=2 ;$ scale bar, $500 \mu \mathrm{m}$ ), 1 month after implantation into the pig omental bursa. I Representative Masson's trichrome (and a higher magnification) staining images of retrieved SB-SLG100 alginate microcapsules $(n=2$; scale bar, $500 \mu \mathrm{m})$. $\mathbf{m}$ PAS-stained histology (and a higher magnification; scale bar, $200 \mu \mathrm{m}$ ) images of retrieved SLG100 microcapsules (Scale bar, $1 \mathrm{~mm}$ ). Arrow indicates foreign body giant cells. $\mathbf{n}$ PAS-stained histology (and a higher magnification; scale bar, $200 \mu \mathrm{m}$ ) images of retrieved SB-SLG100 microcapsules (scale bar, $1 \mathrm{~mm}$ )

90 days, whereas all the mice from the SB-SLG20 group remained normoglycemic for 3 months before the microcapsules were retrieved. We also performed an intraperitoneal glucose tolerance test (IPGTT) (Fig. 5c) 90 days after transplantation, immediately prior to retrieval on selected mice. While three mice with lowest BG levels from the SLG20 control group failed to reduce BG to normoglycemic range even $180 \mathrm{~min}$ after glucose challenge, mice in the SB-SLG20 group $(n=3)$ achieved normoglycemia within $90 \mathrm{~min}$, confirming the improved function of transplanted islets (Fig. 5c). Furthermore, the glucose-stimulated insulin secretion (GSIS) assay performed on the retrieved SB-SLG20 microcapsules (Fig. 5d) showed that encapsulated islets were responsive to glucose change and secreted insulin, further supporting for normal islet function.

Post-retrieval characterizations also showed marked differences between the SB-SLG20 microcapsules and control microcapsules, with the former showing almost no cellular deposition (Fig. 5e and Supplementary Fig. 35). Over 90\% of SB-SLG20 microcapsules fell into the $0-25 \%$ PCO category (Supplementary Fig. 36). In the SB-SLG20 microcapsules, there were numerous rat islets (See Fig. 5e and Supplementary Fig. 35 for all samples $n=6$ from two batches) observed with healthy morphology (H\&E staining in Fig. 5f) and positive insulin staining (Fig. 5g). On the contrary, retrieved SLG20 microcapsules showed a large variation in CO 
a
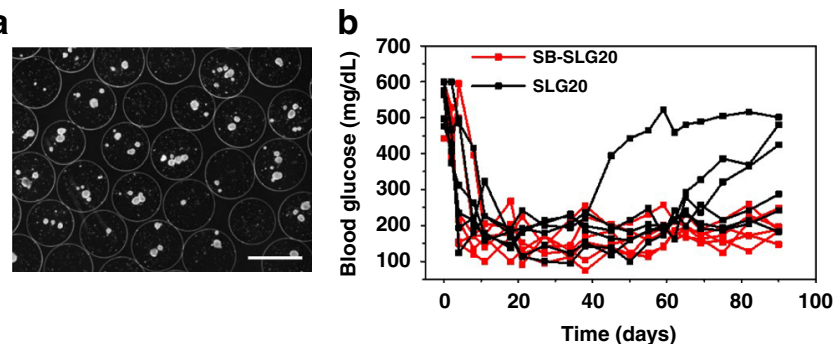

e

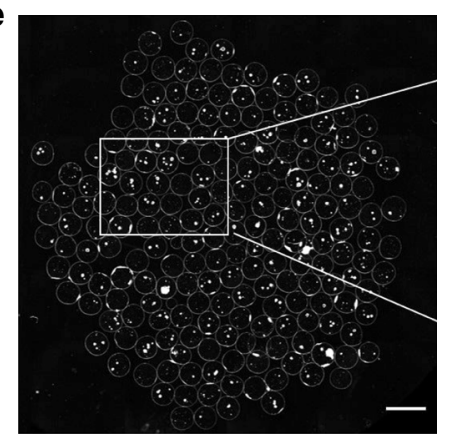

h

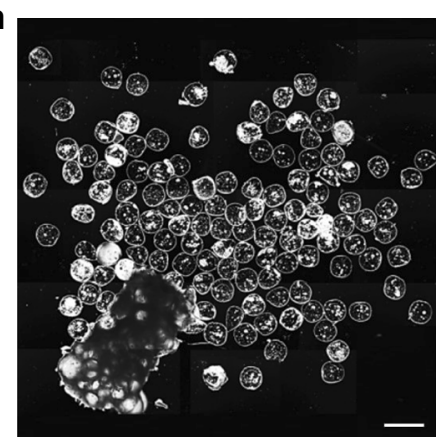

c

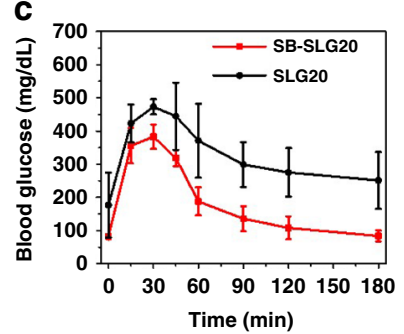

$\mathbf{f}$
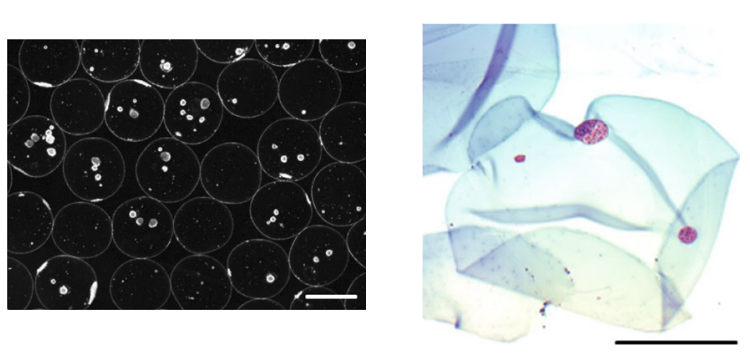

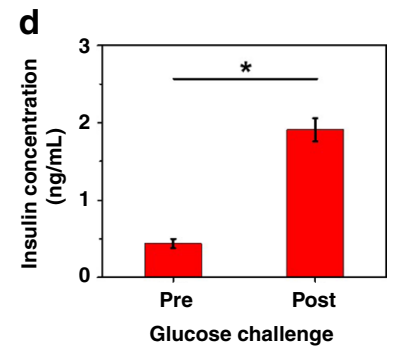

g
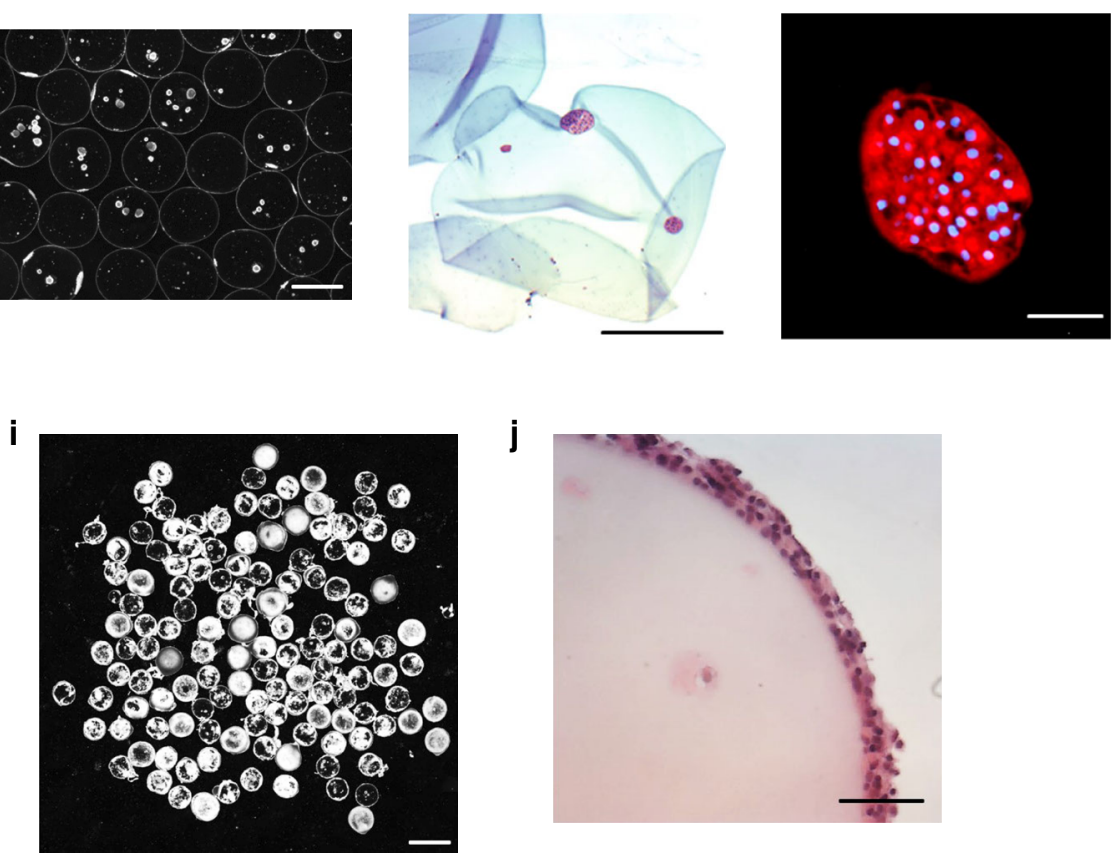

Fig. 5 SB-SLG20 microcapsules improve diabetes correction in mice in a 90-day study. a A dark-field phase contrast image of SLG20 microcapsules encapsulating rat islets before transplantation. Scale bar, $1 \mathrm{~mm}$. b Blood glucose concentrations of mice $(n=6$ per treatment group). c Intraperitoneal glucose tolerance test (IPGTT) before retrieval $(n=3)$. d Ex vivo glucose-stimulated insulin secretion (GSIS) of retrieved islet-containing SB-SLG20 microcapsules, $n=3$, Mean \pm SEM, ${ }^{\star} P<0.05$. e A dark-field phase contrast image of retrieved islet-containing SB-SLG20 microcapsules ( $n=6$; see Supplementary Fig. 35 for complete data. Scale bars, $2 \mathrm{~mm}$ on the left and $1 \mathrm{~mm}$ on the right). $\mathbf{f}$ An H\&E stained cross-sectional image of retrieved isletcontaining SB-SLG20 microcapsules. Scale bar, $500 \mu \mathrm{m}$. $\mathbf{g}$ Immunohistochemical staining of a rat islet in a retrieved SB-SLG20 microcapsule. Insulin is stained red and nuclei are stained blue (Scale bar, $50 \mu \mathrm{m}$ ). $\mathbf{h}$, i Dark-field phase contrast images of retrieved islet-containing SLG20 microcapsules from the normoglycemic mouse group (h) and failed ones (i). $(n=6$; scale bar $=2 \mathrm{~mm}$; see Supplementary Fig. 37 for complete data). j An H\&E stained crosssectional image of retrieved islet-containing SLG20 microcapsule (Scale bar, $200 \mu \mathrm{m}$ )

(See Fig. 5h, Fig. 5i and Supplementary Fig. 37 for all samples $n=$ 6 from two batches). Approximately $19.1 \%$ of all retrieved SLG20 microcapsules were within the $0-25 \%$ PCO range and $48.3 \%$ fell into the $75-100 \%$ PCO category (Supplementary Fig. 36). Approximately $75 \%$ of the SLG20 microcapsules from the 2 normoglycemic mice had moderate to little $\mathrm{CO}$, while the majority of the microcapsules from the 4 failed mice had severe cellular deposition, suggesting a correlation between $\mathrm{CO}$ level and diabetes correction. As expected, the islets in the microcapsules with cellular deposition either exhibited unhealthy morphology or were completely dead as shown by the H\&E staining of cross-sections (Fig. 5j).

To further study the robustness of the SB-alginate in improving islet encapsulation and sustaining normoglycemia, we performed a longer-term, 200-day transplantation experiment. Four out of the six diabetic mice transplanted with rat islets encapsulated in SB-SLG20 microcapsules maintained normoglycemia after 200 days (Fig. 6a); the shortest duration of glycemic control was $\sim 135$ days. However, almost all the mice transplanted with rat islets encapsulated in SLG20 microcapsules returned to hyperglycemia by 100 days after implantation. An IPGTT assay (Fig. 6b) 200 days after transplantation, right before retrieval showed that the mice (cured ones, $n=3$ ) in the SB-SLG20 group cleared $\mathrm{BG}$ and restored normoglycemia at a rate comparable to that of non-diabetic mice, while the BG of the mice $(n=3)$ in the SLG20 control group failed to drop to normal range after 150 min, similar to non-transplanted diabetic mice. For the SB-SLG20 group, an ex vivo GSIS (Fig. 6c) of islets retrieved from cured mice $(n=3)$ indicated again the normal function of islets. Darkfield microscopic images of retrieved SB-SLG20 microcapsules (See Fig. 6d and Supplementary Fig. 38 for all samples $n=6$ from two batches) from normoglycemic mice after 200 days revealed no or minimal cellular deposition on the microcapsules and the presence of numerous islets inside. PCO quantification showed that $81.5 \%$ of SB-SLG20 microcapsules were largely free of CO (Supplementary Fig. 39). More importantly, the retrieved islets were functional, as verified by $\mathrm{H} \& \mathrm{E}$ histological analysis (Fig. 6e) and positive insulin staining (Fig. 6f). In contrast, the SLG20 microcapsules produced severe $\mathrm{CO}$ as shown by dark-field phase contrast microscopic images (See Fig. $6 \mathrm{~g}$ and Supplementary 


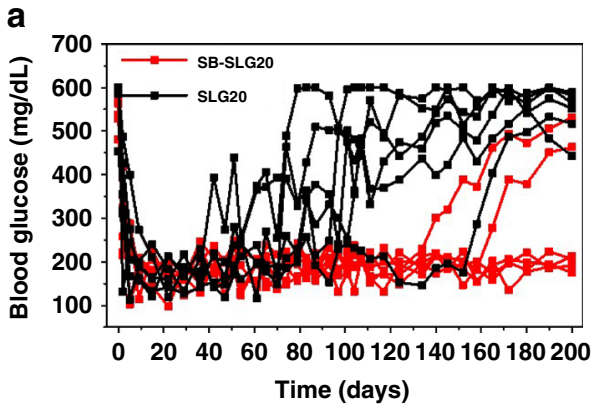

b
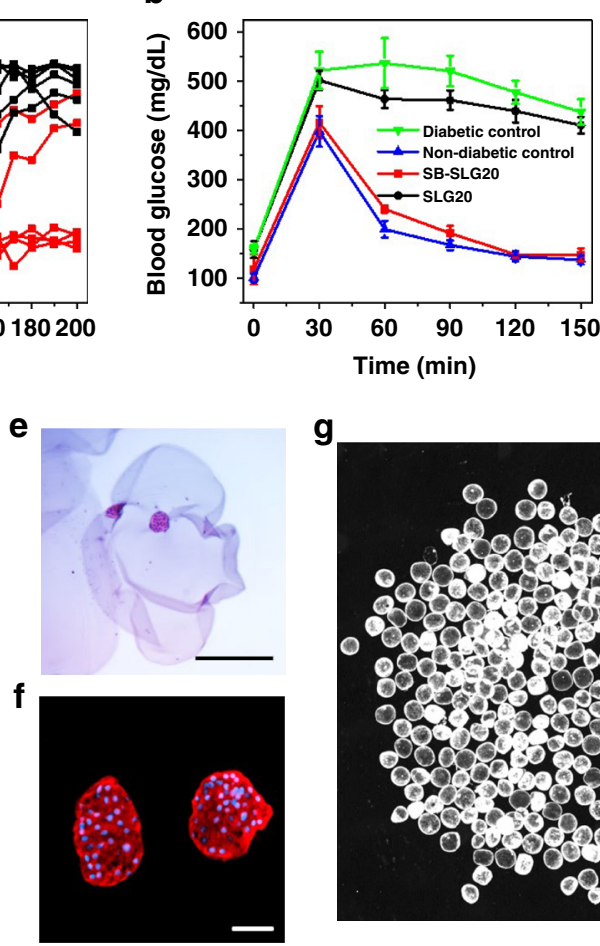

C

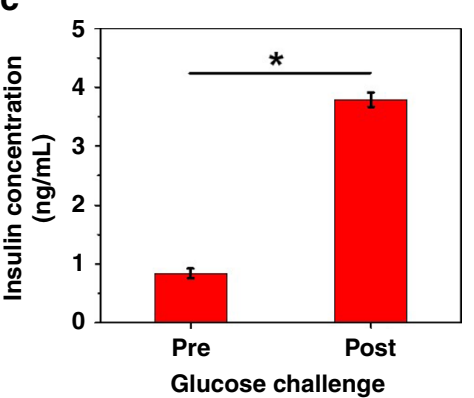

h
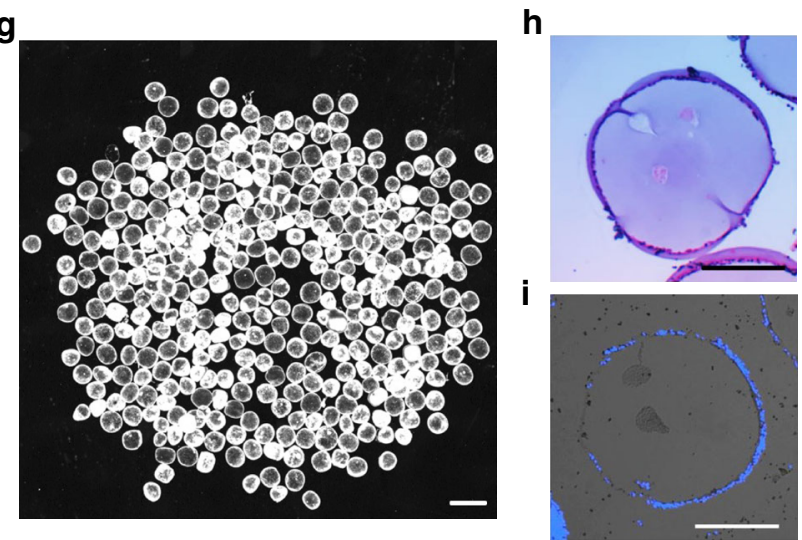

Fig. 6 SB-SLG20 microcapsules improve diabetes correction in mice in a 200-day study. a Blood glucose concentrations of mice ( $n=6$ mice per treatment group). b Intraperitoneal glucose tolerance test (IPGTT) before retrieval $(n=3)$. c Ex vivo GSIS of the retrieved rat islets from SB-SLG20 microcapsules, $n=3$, Mean \pm SEM, ${ }^{\star} P<0.05$. d A dark-field phase contrast image of retrieved islet-containing SB-SLG20 microcapsules. ( $n=6$; scale bar, 2 mm; see Supplementary Fig. 38 for complete data). e An H\&E stained cross-sectional image of retrieved islet-containing SB-SLG20 microcapsules. Scale bar, $500 \mu \mathrm{m}$. f Immunohistochemical staining of rat islets in retrieved SB-SLG20 microcapsules. Insulin is stained red and nuclei are stained blue (Scale bar: $50 \mu \mathrm{m}) . \mathbf{g ~ A}$ dark-field phase contrast image of retrieved islet-containing SLG20 microcapsules. ( $n=6$; scale bar, 2 mm; see Supplementary Fig. 40 for complete data). h An H\&E stained cross-sectional image of retrieved islet-containing SLG20 microcapsules. Scale bar, $500 \mu \mathrm{m}$. i Immunohistochemical staining of rat islets in retrieved SLG20 microcapsules. Insulin staining is negative and nuclei are stained blue. Scale bar, $500 \mu \mathrm{m}$

Fig. 40 for all samples $n=6$ from two batches), by the PCO quantification (Supplementary Fig. 39), and by the H\&E staining (Fig. 6h). The H\&E staining also showed unhealthy or non-viable morphology of encapsulated islets and the immunohistochemical staining of insulin (Fig. 6i) was negative. Furthermore, the retrieval rate for the SB-SLG20 microcapsules was significantly higher than that for the SLG20 microcapsules (Supplementary Table 2). Taken together, the SB modification drastically improved the outcome of islet microencapsulation, achieving long-term glycemic correction for up to 200 days in STZ-treated C57BL/6J mice.

\section{Discussion}

FBR against implanted biomaterials and medical devices represents a major hurdle to many biomedical engineering applications, particularly cell encapsulation. Unfortunately, despite its importance, FBR is an incompletely understood process involving complex biological cascades and high heterogeneity. For alginate microcapsules, which have been used for decades for cell encapsulation, it has been shown that many parameters including types of alginates ${ }^{39,51}$, purity of alginate (presence of endotoxins, proteins, and polyphenols) $)^{39,52}$, alginate compositions ${ }^{53-56}$, microcapsule size $^{41,57}$ and subtle changes in formulations ${ }^{58-62}$ can all affect the degree of FBR or CO. Furthermore, the presence or absence of additional coating layers such as poly-L-lysine or chitosan resulted in varying degrees of $\mathrm{CO}^{63,64}$. The choice of cross-linking ions (usually calcium or barium) was reported to influence inflammatory response against alginate-based capsules $^{65}$. Alginate capsules containing anti-inflammatory drugs has been employed as a strategy to mitigate the $\mathrm{CO}$ and improve the implantation outcome ${ }^{66,67}$. However, reproducibility of microcapsule performance even in mice has been a challenge for the field. Different labs often report different results in terms of $\mathrm{CO}^{19,41,68-70}$. Indeed, we have often observed in our laboratory variations of $\mathrm{CO}$ against the unmodified control microcapsules between experiments, among different animals in the same experiment, and even among different microcapsules within the same animal. In our present study, we made significant efforts to retrieve, image and analyze all microcapsules from all mice. For unmodified control microcapsules, while there always existed a small fraction that were relatively clean, the majority of them had $\mathrm{CO}$ to different degrees. These observations were consistent with those reported recently for microcapsules made of similar alginates with similar dimensions $(<1 \mathrm{~mm})^{41}$. In contrast, for the zwitterionically modified alginates, we observed consistent and uniform reduction (and in some cases elimination) of $\mathrm{CO}$. Additional large-animal experiments showed that the FBRmitigating effect was reproducible across different animal species including $\mathrm{C} 57 \mathrm{BL} / 6 \mathrm{~J}$ mice, dogs and pigs.

Although CO-mitigating, chemically modified alginates have been reported previously, those "hits" were discovered by timeconsuming screenings of a total of 774 different types of chemical modifications ${ }^{23}$. Our zwitterionic modification of alginates represents a simpler and much less expensive approach and led to alginate derivatives that were shown comparable to those obtained by screening. The rationale is based on the well-studied anti-fouling properties of zwitterionic moieties. In this work, we also started to explore the mechanisms of the CO-mitigating 
effect. Our data, consistent with literature ${ }^{34,71}$, supported that zwitterionic groups due to their strong hydration mitigated nonspecific protein adsorption which is a key first step in foreign body responses. Likely as a result of the decreased protein adsorption, the zwitterionic modification altered macrophage activation, TLR2 inhibition and cytokine expression in the peritoneal fluid which might be contributing factors to the observed CO mitigation. Despite these studies, more work is required to elucidate the exact mechanisms.

To demonstrate and confirm the CO-mitigating effect of zwitterionic modification, we modified three different ultrapure, sterile alginates (VLVG, SLG20 and SLG100) and used three different zwitterions, a SB and two CBs. All the zwitterionically modified alginates (SB-alginates, $\mathrm{CB} 1$-alginate and $\mathrm{CB} 2$-alginate) were shown to mitigate $\mathrm{CO}$ compared to the unmodified control. Incorporating a zwitterionic moiety into alginate therefore opens up a new avenue for the design and development of superbiocompatible alginates.

The therapeutic potential of the SB modified alginate was explored through a type 1 diabetic mouse model using rat islets. Even in the presence of rat islets (i.e., xenogeneic tissue), the SBSLG20 microcapsules had no or minimal CO in C57BL/6J mice, while the unmodified, control SLG20 microcapsules were mostly covered by $\mathrm{CO}$. The reduced $\mathrm{CO}$ correlated with an improved outcome for islet encapsulation and transplantation. The SBSLG20 microcapsules encapsulating rat islets enabled longer and more robust correction of STZ-induced diabetes in C57BL/6J mice. A 200-day cure was achieved for four out of six mice using SB-SLG20 microcapsules. Moreover, the ability of zwitterionic modification to mitigate FBR was translatable to higher-order species (dogs and pigs) and in different implantation sites (intraperitoneal cavity and omental bursa), suggesting great potential for future clinical applications. Zwitterionic materials have been used for a number of applications including fabrication of antifouling surfaces, grafting of implantable devices and biosensors, and formation of drug delivery micelles and nanogels $26,28,72-77$. Here, we report the use of zwitterionically modified materials for cell encapsulation for potential T1D treatment. This approach may contribute to a cell replacement therapy for not only T1D but also other hormone-deficient diseases such as hemophilia.

\section{Methods \\ Study design. The aim of this study was to explore whether zwitterionically modified alginates could mitigate $\mathrm{CO}$ reproducibly at various implantation time points and across different species. To test this, all experiments using C57BL/6J mice, Sprague Dawley rats, and Beagle dogs were conducted at Cornell University, approved by the Cornell Institutional Animal Care and Use Committee, and car- ried out by trained personnel. Transplantation of alginate microcapsules in insulin treated type 1 STZ-induced Göttingen minipigs was performed at Novo Nordisk A/ $\mathrm{S}$, and the protocols were approved by the Danish Animal Experimentation Inspectorate and carried out by trained and licensed personnel. Alginate micro- capsules were retrieved and imaged, and histopathology using H\&E as well as special stains was performed by trained individuals.}

Materials/reagents. Di-tert-butyl dicarbonate, triethylamine, $\mathrm{N}, \mathrm{N}$-Dimethylethylenediamine, $\beta$-Propiolactone, tert-butyl bromoacetate, barium chloride, magnesium sulfate, magnesium chloride hexahydrate, phosphate-buffered saline (PBS; pH 7.4, $10 \mathrm{mM}, 138 \mathrm{mM} \mathrm{NaCl}, 2.7 \mathrm{mM} \mathrm{KCl}$ ), HEPES buffer, diethyl ether, ethyl alcohol, acetonitrile and dichloromethane (DCM) were obtained from SigmaAldrich. 2-chloro-4, 6-dimethoxy-1, 3, 5-triazine (CDMT), $N$-methylmorpholine (NMM), 1, 3-propanesultone and trifluoroacetic acid (TFA) were purchased from the Alfa Aesar. All the sodium alginates including VLVG ( $>60 \% \mathrm{G}, 25 \mathrm{kDa} \mathrm{MW})$, SLG20 (>60\% G, 75-220 kDa MW), and SLG100 (>60\% G, 200-300 kDa MW), were purchased from FMC BioPolymer Co. (Philadelphia, PA). Cyano-

functionalized silica was purchased from SiliCycle. Rabbit anti-insulin antibodies (Cat. \#ab63820) was purchased from Abcam, and Alexa Fluor 594-conjuaged donkey anti-rabbit igG (Cat. \#A-21207) was purchased from Invitrogen. $a$-smooth muscle actin (SMA) (Cat. \#C6198) was purchased from Sigma-Aldrich, and antimouse CD68 (Cat. \#137012) was purchased from BioLegend. Anti-mouse F4/80
(Cat. MF48000) was purchased from ThermoFisher, and anti-CD11b (Cat. \#ab133357) was purchased from Abcam. Anti-mouse Ly-6G/Ly-6C (Cat. \#108419) was purchased from BioLegend. Proteome profiler array kit (Mouse Cytokine Array Panel A; Cat. \#ARY006) was purchased from R\&D Systems.

Synthesis of SB-based conjugates. Synthesis of SB-NH $\mathrm{N}_{2}$ material is shown in Supplementary Fig. 1. Briefly, di-tert-butyl dicarbonate $(10.0 \mathrm{~g}, 45.8 \mathrm{mmol})$ and triethylamine $(12.8 \mathrm{~mL}, 91.6 \mathrm{mmol})$ were added dropwise over $0.5 \mathrm{~h}$ to a solution of $\mathrm{N}, \mathrm{N}$-Dimethylethylenediamine $(4.04 \mathrm{~g}, 45.8 \mathrm{mmol})$ in anhydrous ethyl alcohol $(150 \mathrm{~mL})$ at $0{ }^{\circ} \mathrm{C}$. The mixture was stirred for $1 \mathrm{~h}$ at $0{ }^{\circ} \mathrm{C}$ and then for $18 \mathrm{~h}$ at room temperature. The white precipitate was filtered off and the filtrate was evaporated to obtain residue. The residue was dissolved in dichloromethane $(150 \mathrm{~mL})$, and the solution was washed successively with water. The organic layer was dried over anhydrous magnesium sulfate and evaporated to get $N, N$-dimethyl-2-((pivaloyloxy) amino) ethan-1-amine. ${ }^{1} \mathrm{H}$ NMR $\left(\mathrm{CDCl}_{3}, 400 \mathrm{MHz}\right): \delta 3.22(\mathrm{t}, 2 \mathrm{H}), 2.29(\mathrm{t}$, $2 \mathrm{H}), 2.22(\mathrm{~s}, 6 \mathrm{H}), 1.43(\mathrm{~s}, 9 \mathrm{H})$.

$\mathrm{N}, \mathrm{N}$-dimethyl-2-((pivaloyloxy) amino) ethan-1-amine (7.52 g, $40.0 \mathrm{mmol}), 1$, 3-propanesultone $(4.9 \mathrm{~g}, 40.0 \mathrm{mmol})$ and acetonitrile $(150 \mathrm{~mL})$ were added into a $300 \mathrm{~mL}$ round-bottom flask. The mixture was stirred under nitrogen atmosphere for $48 \mathrm{~h}$ at $40^{\circ} \mathrm{C}$. After reaction, the solvent was removed by rotary evaporator. The product was precipitated by anhydrous diethyl ether and washed with anhydrous diethyl ether to get white powder. ${ }^{1} \mathrm{H}$ NMR $\left(\mathrm{D}_{2} \mathrm{O}, 400 \mathrm{MHz}\right): \delta 3.41-3.63(\mathrm{~m}, 6 \mathrm{H})$, $3.17(\mathrm{~s}, 6 \mathrm{H}), 2.98(\mathrm{~m}, 2 \mathrm{H}), 2.24(\mathrm{~m}, 2 \mathrm{H}), 1.43(\mathrm{~s}, 9 \mathrm{H})$.

Finally, $10.0 \mathrm{~g}$ of the obtained product was treated with a mixture of $20 \mathrm{~mL}$ trifluoroacetic acid (TFA) and $20 \mathrm{~mL}$ dichloromethane overnight at room temperature, concentrated with rotary evaporator, precipitated in anhydrous diethyl ether, and re-dissolved in DI water. Ion-exchange resin (Amberlyst A26, $\mathrm{OH}$-form) was added into the solution for complete neutralization. The residue was lyophilized by freeze dryer to obtain product (SB-NH $\mathrm{NH}_{2}$ material). ${ }^{1} \mathrm{H}$ NMR $\left(\mathrm{D}_{2} \mathrm{O}, 400 \mathrm{MHz}\right): \delta 3.70(\mathrm{t}, 2 \mathrm{H}), 3.54(\mathrm{~m}, 4 \mathrm{H}), 3.20(\mathrm{~s}, 6 \mathrm{H}), 2.97(\mathrm{t}, 2 \mathrm{H}), 2.24$ $(\mathrm{m}, 2 \mathrm{H})$.

Synthesis of SB-modified alginate: $0.5 \mathrm{~g}$ of VLVG alginate, SLG20 alginate or SLG100 alginate was soluble in $50 \mathrm{~mL}$ mixture solvent $(40 \mathrm{~mL}$ of DI water and $10 \mathrm{~mL}$ acetonitrile). $225 \mathrm{mg}$ of 2-chloro-4, 6-dimethoxy-1, 3, 5-triazine (CDMT) and $280 \mu \mathrm{L}$ of $\mathrm{N}$-methylmorpholine (NMM) were added. Then $0.36 \mathrm{~g}$ of SB-NH material was dissolved in $10 \mathrm{~mL}$ DI water and added into the mixture. The reaction was stirred overnight at $60^{\circ} \mathrm{C}$. The solvent was removed under reduced pressure and the solid product was redissolved in DI water. The solution was filtered through a pad of cyano-functionalized silica. It was then dialyzed against a 10,000 MWCO membrane in DI water for three days. Finally, the water was removed by freeze dryer to obtain SB-modified alginate. ${ }^{1} \mathrm{H}$ NMR $\left(\mathrm{D}_{2} \mathrm{O}, 400 \mathrm{MHz}\right): \delta 3.5-5.3$ (m, alginate protons), $3.87(\mathrm{~m}, 2 \mathrm{H}), 3.61(\mathrm{~m}, 4 \mathrm{H}), 3.14(\mathrm{~s}, 6 \mathrm{H}), 2.91(\mathrm{t}, 2 \mathrm{H}), 2.20$ $(\mathrm{m}, 2 \mathrm{H})$. There was about $30.5 \%$ modification degree of the starting alginate through NMR analysis.

Synthesis of CB-based conjugates. Synthesis of CB1- $\mathrm{NH}_{2}$ materials is shown in Supplementary Fig. 8. Briefly, N, N-dimethyl-2-((pivaloyloxy)amino)ethan-1amine $(7.52 \mathrm{~g}, 40.0 \mathrm{mmol})$, tert-butyl bromoacetate $(7.8 \mathrm{~g}, 40.0 \mathrm{mmol})$ and acetonitrile $(150 \mathrm{~mL})$ were added into a $250 \mathrm{~mL}$ round-bottom flask. The mixture was stirred under nitrogen atmosphere for $48 \mathrm{~h}$ at $40^{\circ} \mathrm{C}$. After reaction, the solvent was removed by rotary evaporator. The product was precipitated by anhydrous diethyl ether and washed with anhydrous diethyl ether three times to get white powder. ${ }^{1} \mathrm{H}$ NMR $\left(\mathrm{D}_{2} \mathrm{O}, 400 \mathrm{MHz}\right): \delta 4.21(\mathrm{~s}, 2 \mathrm{H}), 3.72(\mathrm{~m}, 2 \mathrm{H}), 3.57(\mathrm{~m}, 2 \mathrm{H}), 3.31(\mathrm{~s}, 6 \mathrm{H})$, $1.32-1.54(\mathrm{~s}, 18 \mathrm{H})$

Finally, $5.0 \mathrm{~g}$ of the obtained product was treated with a mixture of $20 \mathrm{~mL}$ trifluoroacetic acid and $20 \mathrm{~mL}$ dichloromethane overnight at room temperature, concentrated with rotary evaporator, precipitated in anhydrous diethyl ether, and redissolved in DI water. Ion-exchange resin (Amberlyst A26, OH-form) was added into the solution for complete neutralization. The residue was lyophilized by freeze dryer to obtain product (CB1- $\mathrm{NH}_{2}$ monomer). ${ }^{1} \mathrm{H}$ NMR $\left(\mathrm{D}_{2} \mathrm{O}, 400 \mathrm{MHz}\right): \delta 4.31$ $(\mathrm{s}, 2 \mathrm{H}), 3.99(\mathrm{t}, 2 \mathrm{H}), 3.55(\mathrm{t}, 2 \mathrm{H}), 3.36(\mathrm{~s}, 6 \mathrm{H})$. The $\mathrm{CB} 2-\mathrm{NH}_{2}$ monomer as shown in Supplementary Fig. 9 was synthesized using the same procedure as that for CB-1$\mathrm{NH}_{2}$ monomer. ${ }^{1} \mathrm{H}$ NMR of CB2- $\mathrm{NH}_{2}\left(\mathrm{D}_{2} \mathrm{O}, 400 \mathrm{MHz}\right): \delta 3.63-3.75(\mathrm{~m}, 4 \mathrm{H}), 3.53$ $(\mathrm{m}, 2 \mathrm{H}), 3.18(\mathrm{~s}, 6 \mathrm{H}), 2.96(\mathrm{t}, 2 \mathrm{H})$.

Synthesis of CB-modified alginates: The CB1-alginate and CB2-algiante conjugates were synthesized using the same procedure as that for SB-alginate conjugate. The chemical structures of these CB-based alginate conjugates were confirmed by NMR. ${ }^{1} \mathrm{H}$ NMR of CB1-alginate: $\delta=3.5-5.3$ (m, alginate protons), $3.84-4.10(\mathrm{~m}, 4 \mathrm{H}), 3.72(\mathrm{~m}, 2 \mathrm{H}), 3.34(\mathrm{~s}, 6 \mathrm{H})$. There was about $33.1 \%$ modification degree of the starting alginate through the NMR analysis. ${ }^{1} \mathrm{H}$ NMR of CB2alginate: $\delta=3.5-5.3(\mathrm{~m}$, alginate protons), 3.87-4.06 $(\mathrm{m}, 4 \mathrm{H}), 3.80(\mathrm{~m}, 2 \mathrm{H}), 3.39(\mathrm{~s}$ $6 \mathrm{H}), 2.95(\mathrm{~m}, 2 \mathrm{H})$. There was about $24.7 \%$ modification degree of the starting alginate through NMR analysis.

Preparation of SB-based or CB-based alginate microcapsules. All the buffers were sterilized, and alginate solutions were filtered using a $0.2 \mu \mathrm{m}$ filter before use $2 \%(\mathrm{w} / \mathrm{v})$ alginate (VLVG, SLG20, or SLG100) was dissolved in saline solution to prepare an alginate solution. $2 \%(\mathrm{w} / \mathrm{v}) \mathrm{SB}$-based alginate conjugate was dissolved in saline solution. The mixture of $60 \%$ (by volume) SB-based alginate solution and 
$40 \%$ (by volume) SLG100 solution were blended to obtain the SB-alginate solution. The CB-alginate solution was prepared using the same procedure.

Alginate hydrogel microcapsules were made using a custom-built electrospraying setup. Briefly, a high voltage power generator was connected to a blunt-tipped needle. This needle was attached to a $3 \mathrm{~mL}$ syringe containing alginate solution clipped to a vertically oriented syringe pump. Various sizes of microcapsules were prepared using different voltage, flow rate and needle gauges; $500-700 \mu \mathrm{m}$ microcapsules were prepared using a voltage of $8-9 \mathrm{kV}$, a $0.2 \mathrm{~mL} / \mathrm{min}$ flow rate and a 25-gauge blunt needle (SAI Infusion Technologies), $800-1000 \mu \mathrm{m}$ microcapsules were prepared using a voltage of $6-7 \mathrm{kV}$, a $0.2 \mathrm{~mL} / \mathrm{min}$ flow rate and a 20-gauge blunt needle (SAI Infusion Technologies). Alginate solution was sprayed into a sterile, grounded dish containing a $20 \mathrm{mM}$ barium chloride solution. Once the alginate microcapsules were fabricated, they were collected immediately and washed with prepared HEPES buffer $\left[\mathrm{NaCl} 15.43 \mathrm{~g}, \mathrm{KCl} 0.70 \mathrm{~g}, \mathrm{MgCl}_{2} \bullet 6 \mathrm{H}_{2} \mathrm{O}\right.$ $0.49 \mathrm{~g}, 50 \mathrm{~mL}$ of HEPES $(1 \mathrm{M})$ buffer solution in $2 \mathrm{~L}$ of DI water] three times and then washed additional two times with saline and kept at $4{ }^{\circ} \mathrm{C}$ before use.

Protein adsorption. Two percent of SB modified alginate solution and two percent of SLG100 alginate solution were mixed at a 60:40 (v/v) ratio. Two percent of SLG20 was used as control. $0.2 \mathrm{~mL}$ of the above alginate solutions were added to a 24-well plate. Then, $0.2 \mathrm{~mL}$ of barium chloride solution $(20 \mathrm{mM})$ as a cross-linking buffer was slowly added on the top of the gel. After $1 \mathrm{~h}$ solidification, these alginate hydrogels were removed from plates and washed with PBS five times before use.

The above alginate hydrogels were immersed in FITC-labeled fibrinogen solution $(0.1 \mathrm{mg} / \mathrm{mL}$ in PBS) or FITC-labeled lysozyme solution $(0.1 \mathrm{mg} / \mathrm{mL}$ in PBS) at room temperature for $1 \mathrm{~h}$ to allow protein adsorption on the hydrogel surfaces. Hydrogels were then gently washed with PBS five times to remove the unbound protein molecules. Fluorescence images of hydrogel surfaces were taken using a fluorescence microscope with $10 \times$ lens at a fixed exposure time. To ensure that all hydrogel samples were focused on the same plane, all images were taken at the edge of the hydrogels. The fluorescent intensity was quantified and analyzed using ImageJ software.

Mechanical strength of microcapsules. The mechanical properties of different alginate microcapsules was tested with a Texture Analyzer XT plus (Stable Micro Systems, Godalming, UK) equipped with a force transducer (Resolution: $1 \mathrm{mN}$ ). Microcapsules were carefully monitored under a dissection microscope (Bausch and Lomb BVB-125). Uniaxial compression tests were performed as follows: a mobile probe $(\mathrm{P} / 25 \mathrm{~L})$ with pretest speed of $0.5 \mathrm{~mm} / \mathrm{s}$, a test speed of $0.01 \mathrm{~mm} / \mathrm{s}$, and a post-test speed of $2 \mathrm{~mm} / \mathrm{s}$ was used to compress individual microcapsules $(n=10)$ with a trigger force of two grams. The force (grams) was quantified at a $60 \%$ compression of the sample. Resulting measurements were analyzed via Texture Exponent software (v6.0)

Mass transfer studies. To measure the diffusion rate of the unmodified and modified alginate hydrogels, we immersed SLG20 and SB-SLG20 hydrogels into three different saline solutions containing 10,70 , and $250 \mathrm{kDa}$ FITC-labeled dextrans $(1 \mathrm{mg} / \mathrm{mL})$, respectively. The fluorescent intensity of the alginate hydrogel was analyzed using confocal microscopy.

Surface roughness of capsules. Single capsules were carefully selected using a dissection microscope (Leica MZ75 microsystems), washed and placed on microscopic slides. The surface roughness of different microcapsules was determined by a Bruker Catalist atomic force microscope (AFM) with a Bruker DNP silicon nitride cantilever. Topographic imaging of the capsules was performed at room temperature using the contact mode. Surface roughness was calculated by using the NanoScope Analysis 1.8 software.

Zeta potential measurement. Unmodified and modified alginate nanogels were prepared via a surfactant-free and organic solvent-free method ${ }^{78}$. Briefly, sodium alginate was mixed with ionic calcium by in situ crosslinking under ultrasound, and alginate nanogels were obtained. Zeta potentials of alginate nanogels were then investigated using Zetasizer Nano ZS (Malvern, U.K.). The value was recorded as the mean of five measurements.

TLR signaling. To study whether zwitterionic modified alginate alters TLR signaling, we performed the HEK-Blue ${ }^{\mathrm{TM}}$ TLR cell-based assays (InvivoGen, France) according to the manufacturer's protocol. These HEK-Blue ${ }^{\mathrm{TM}}$ cells express the Soluble Embryonic Alkaline Phosphatase (SEAP) gene which can be quantified using Quantiblue (InvivoGen, France) ${ }^{39,79}$. The SEAP gene is under the control of the NFkB/AP-1 responsive promoter. The information of cell lines, antibiotics, and concentration of agonists used to activate TLR signaling were shown in Supplementary Table 3. All cell lines were cultured in DMEM culture media (Lonza, Basel, Switzerland) supplemented with $10 \%$ de-complemented Fetal Calf serum, 50 $\mathrm{U} / \mathrm{ml}$ Penicillin (Sigma, St. Louis, MO, USA), $50 \mu \mathrm{g} / \mathrm{ml}$ Streptomycin (Sigma, St. Louis, MO, USA) and $100 \mu \mathrm{g} / \mathrm{ml}$ Normocin (InvivoGen, Toulouse, France). HEKBlue $^{\mathrm{TM}}$ cell lines for TLR2 expressing cells at a density of $2.8 \times 10^{5} \mathrm{cells} / \mathrm{ml}$ and
HEK- Blue ${ }^{\mathrm{TM}}$ cell lines for TLR4 expressing cells at a density of $1.4 \times 10^{5}$ cells $/ \mathrm{ml}$ were seeded in 96 -well plates at $180 \mu \mathrm{l} /$ well, respectively. After overnight culture, Cells were treated with 25 alginate capsules or TLR agonists as control to study whether alginate capsules can activate TLR signaling (Supplementary Table 3). Inhibition of TLR was studied by exposing HEK-Blue ${ }^{\mathrm{TM}}$ cells with 25 alginate capsules for $1 \mathrm{~h}$, followed by treatment with TLR agonists (Supplementary Table 3). QUANTI-Blue $^{\text {sut }}$ medium (InvivoGen, Toulouse, France) change to a purple-blue color in the presence of SEAP. Media supernatant from the stimulated cell-lines was mixed with QUANTI-Blue ${ }^{\mathrm{m} w}$ medium in a ratio of 1:10 (v/v). Activation of NFkB was finally quantified at $655 \mathrm{~nm}$ using a Versa Max ELISA plate reader (Molecular devices, Sunnyvale, CA, USA). The assay was performed with six repeats.

Cytokine secretion. All animal procedures were approved by the Institutional Animal Care and Use Committee at the University of California Irvine prior to initiation of the study. BMDM were harvested from the femurs or tibia of 6-8 week-old C57BL/6J mice (Jackson Laboratories). Cells were treated with ACK lysis buffer (Invitrogen), centrifuged, and resuspended in D-10 media. D-10 media consists of Dulbecco's modified Eagle medium (DMEM), 10\% heat-inactivated FBS, $2 \mathrm{mM}$ L-glutamine, 100 units per $\mathrm{mL}$ penicillin-streptomycin, and $10 \%$ conditioned media from CMG 14-12 cells expressing macrophage colony stimulating factor $(\mathrm{M}-\mathrm{CSF})^{80}$. After culture for one week, BMDM were dissociated using cell dissociation buffer (Invitrogen), and seeded on the tissue culture plates at a cell density of 100,000 cells $/ \mathrm{cm}^{2}$ (Olympus Plastics) in fresh D-10 culture media. At $6 \mathrm{~h}$ culture after cell seeding, $10 \%$ of $500 \mu \mathrm{m}$ various alginate capsules $(\mathrm{v} / \mathrm{v})$ were added and stimulated with a combination of $0.3 \mathrm{ng} / \mathrm{mL}$ lipopolysaccharide (LPS) and $1.0 \mathrm{ng} / \mathrm{mL}$ inter-feron gamma (IFN $\gamma$ ). After $12 \mathrm{~h}$ of incubation, the supernatants were collected and analyzed for TNF- $\alpha$ secretion by enzyme-linked immunosorbent assay (ELISA) following the manufacturer's instructions (BioLegend).

Rat islet isolation, purification, and encapsulation. Male Sprague-Dawley rats from Charles River Laboratories weighing about $300 \mathrm{~g}$ were used for harvesting islets. All rats were anesthetized using 3\% isoflurane in oxygen throughout the whole procedure. Isolation surgeries were performed following by Lacy and Kostianovsky ${ }^{81}$. Briefly, the port vein was clamped and rat bile duct was cannulated. The pancreas was distended by an in vivo injection of cold $0.15 \%$ Liberase (Research Grade, Roche) in RPMI 1640 media solution. The perfused pancreatic organs were removed and put into $50 \mathrm{~mL}$ conical tubes on ice until the end of all surgeries. All the tubes containing the pancreas were then placed into in a $37^{\circ} \mathrm{C}$ water bath for a $29 \mathrm{~min}$ digestion. After that, the digestion was quenched by adding cold M199 media containing 10\% heat-inactivated fetal bovine serum (HIFBS) and lightly shaking. Digested pancreases were washed twice by the same aforementioned M199 media, filtered through a $450 \mathrm{~mm}$ sieve, and then suspended in a Histopaque 1077 (Sigma)/M199 media gradient and centrifuged at 1700 RCF at $4{ }^{\circ} \mathrm{C}$. This gradient centrifugation step was repeated for higher purity islets. Finally, these islets were further isolated by a series of six gravity sedimentations, in which each supernatant was discarded after 4 min of settling. Purified islets were handpicked under the microscope and washed by sterile saline solution. Islets were then cultured overnight in RPMI 1640 media with 10\% HIFBS and 1\% penicillin/ streptomycin for further use.

Prior to islet encapsulation, the cultured islets were centrifuged at 562 RCF for $1 \mathrm{~min}$ and washed with saline solution. After washing, islets were centrifuged again and all supernatant was removed. The islet pellet was then resuspended in a $2 \%$ alginate solution at an islet density of 500 islets. $800-1000 \mu \mathrm{m}$ alginate microcapsules containing islets were crosslinked using a $\mathrm{BaCl}_{2}$ gelling solution and their sizes of microcapsules were controlled using similar procedures as the empty spheres (described above). After crosslinking, microcapsules with encapsulated islets were immediately washed five times with saline solution to remove residual $\mathrm{BaCl}_{2}$ gelling solution, transferred into corresponding cell culture medium, and cultured $4 \mathrm{~h}$ at $37^{\circ} \mathrm{C}$ prior to implantation. Since the rat islets had variable sizes $(50-300 \mu \mathrm{m})$ and also there was an inevitable loss of islets during the process of encapsulation, the total number of encapsulated islets was converted into islet equivalents (IE, normalized to $150 \mu \mathrm{m}$ size) following a previously published method $^{82}$.

Implantation and retrieval in mice. Immuno-competent male C57BL/6J mice were used for transplantation. To create insulin-dependent diabetic mice, healthy C57BL $/ 6 \mathrm{~J}$ mice were treated $(50 \mathrm{mg} / \mathrm{kg}$ mouse) with freshly prepared streptozocin (STZ) (Sigma Aldrich) solution $(7.5 \mathrm{mg} / \mathrm{mL}$ in sodium citrate buffer solution) for 5 consecutive days. The BG levels of all the mice were monitored before transplantation. The mice whose non-fasted BG levels of the mice were above $300 \mathrm{mg} /$ $\mathrm{dL}$, were considered to be diabetic. All the mice were anesthetized using $3 \%$ isoflurane in oxygen and maintained at the same rate throughout the procedure. Prior to transplantation, all mice were injected a $0.05 \mathrm{mg} / \mathrm{kg}$ dose of buprenorphine subcutaneously as a pre-surgical analgesic, and their abdomens were shaved and sterilized using betadine and isopropyl alcohol. A $0.5 \mathrm{~mm}$ incision was made along the midline of the abdomen and the peritoneal lining was exposed using blunt dissection. The peritoneal wall was then grasped with forceps, and a 1-mm incision was made along the linea alba. A desired volume of empty microcapsules or 
islets-containing microcapsules loaded into a sterile pipette were implanted into the peritoneal cavity through the incision. The incision was closed using 5-0 taper tipped polydioxanone (PDS II) absorbable sutures. The skin was then closed over the incision using a wound clip.

The empty microcapsules (or with encapsulated rat islets) were retrieved from mice at desired time points post-implantation. Mice were euthanized by $\mathrm{CO}_{2}$ administration followed by cervical dislocation, and larger $(\sim 3 \mathrm{~mm})$ incisions were made in the skin and the peritoneum. $100 \mathrm{mM} \mathrm{CaCl}$ solution was injected into the abdominal cavity repeatedly and washed out all material microcapsules from the abdomen. In certain instances, the microcapsules needed to be retrieved manually if fibrosed directly to intraperitoneal tissues. The retrieved microcapsules were then submerged into a cross-linking solution containing $100 \mathrm{mM} \mathrm{CaCl}_{2}$ for $\sim 1 \mathrm{~min}$ (It is noted that the size of capsules became slightly smaller after retrieval especially for long-term studies possibly due to change of environment from body fluid to a $\mathrm{Ca}^{2+}$-containing stabilizing buffer that was used to collect the microcapsules), and transferred into $4 \%$ paraformaldehyde for fixation or cell culture medium. Lastly, the microcapsules were collected and stacked together into Histogel for histological sectioning.

Implantation and retrieval in dogs. Dogs (Beagle dogs from Marshall Bioresources, Clyde, NY) were premedicated with gylcopyrolate and butorphanol, induced with propofol, and anesthetized using isoflurane and oxygen throughout the surgery. The abdomen was shaved and prepared for sterile surgery. A $10-\mathrm{mm}$ laparoscopic camera port and two 5-mm instrument ports were percutaneously inserted into the abdomen. The abdomen was insufflated to $12 \mathrm{~mm} \mathrm{Hg}$ pressure with $\mathrm{CO}_{2}$. Prior to transplantation, about $10 \mathrm{~mL}$ of prepared microcapsules (size: $500 \mu \mathrm{m})$ and $30 \mathrm{~mL}$ saline solution were loaded into the $60 \mathrm{~mL}$ sterilized syringe. This syringe was then connected with one side of 16-gauge sterile catheter. This flexible catheter was inserted into the left-side instrument port and used to distribute the capsules throughout the abdominal cavity. The alginate capsule solution was gently infused at a rate no greater than $30 \mathrm{~mL} / \mathrm{min}$. A laparoscopic probe was inserted through the right-sided 5-mm port. After implantation, the abdomen was then desufflated and the remaining parts were removed. The port sites were closed with 3-0 polydioxinone suture material and tissue glue.

The retrieval procedure of microcapsules was conducted using the similar method described for implantation. The implanted capsules were firstly located and photographed. About $100 \mathrm{~mL}$ of sterile pre-warmed saline solution was injected to gently wash the peritoneal cavity and then gently aspirated back into the syringe. Closure was conducted with the same method described for implantation.

Implantation and retrieval in pigs. Four female Göttingen minipigs (Ellegaard Minipigs, Dalmose, Denmark) were acclimatized for three weeks. Following acclimatization, the animals were given $60 \mathrm{mg} / \mathrm{kg}$ streptozotocin on three consecutive days to induce a type 1 diabetic phenotype. The animals were subsequently titrated in on long acting exogenous insulin using QD dosing to achieve a target a plasma glucose $\sim 10-15 \mathrm{mM}$. The animals were anaesthetized and underwent a surgical laparotomy performed lege artis. Briefly, the animals were placed in the dorsal recumbency and the surgical area prepared aseptically, a $6 \mathrm{~cm}$ incision was made from processus xiphoideus and extended the in the caudal direction. The stomach was identified and the greater omentum (bursa omentalis) was advanced to the incision site. The visceral and parietal sheet of the omentum was separate and a $3 \mathrm{~cm}$ incision was made in the parietal sheet. Approximately 30,000 microcapsules (capsule size: $500-700 \mu \mathrm{m}$ ) suspended in $50 \mathrm{~mL}$ of sterile saline solution were administered into the omentum. The surgical incision was closed lege artis and the pigs were provided with antibiotic prophylaxis. After 1-month implantation, the pigs were euthanized with a lethal overdose of euthanal, a full necropsy was performed and omentum with embeded microcapsules extracted for histopathology.

Imaging of the retrieved alginate microcapsules. For phase contrast imaging, retrieved alginate microcapsules were gently washed using saline solution and transferred into Petri dishes for phase contrast microscopy using an EVOS AMF4300 imaging system.

Blood glucose monitoring. Starting post-transplantation, a small drop of blood was collected from the tail vein of each mouse using a lancet and tested using a commercial glucometer (Clarity One, Clarity Diagnostic Test Group), approximately three times a week. Mice with unfasted glucose levels below $200 \mathrm{mg} / \mathrm{dL}$ were considered normoglycemic.

IPGTT assay. Prior to retrieval, glucose tolerance tests were performed to assess metabolic capacity. Mice were fasted overnight before an i.p. injection of glucose solution ( $2 \mathrm{~g}$ of glucose per $1 \mathrm{~kg}$ of body mass). BG levels were monitored at predetermined time points $(0,15,30,60,90,120$, and $180 \mathrm{~min})$ after injection.
$\mathrm{NaHCO}_{3}$ (all from Sigma-Aldrich) supplemented with $20 \mathrm{mM} \mathrm{HEPES}$ and $0.1 \% \%$ BSA (Serological)] was prepared beforehand. Encapsulated islets were then incubated for $60 \mathrm{~min}$ with $2.8 \mathrm{mM}$ or $16.7 \mathrm{mM} \mathrm{d}$-glucose under the same condition. The supernatants were collected, and insulin content was quantified using ultrasensitive mouse/rat insulin ELISA kit (ALPCO) with measurement by Synergy 4 Fluorescence Absorbance Microplate Reader (BioTek) at $450 \mathrm{~nm}$ wavelengths. All of the ELISA results were normalized to the IEQs.

Cytokine profiling analysis. Semi-quantitative analysis of cytokine/chemokine levels was performed using Proteome Profiler ${ }^{\mathrm{TM}}$ Array, Mouse Cytokine Array Panel A (ARY006; R\&D Systems) according to manufacturer's instructions. Briefly, $500 \mu \mathrm{l}$ of intraperitoneal liquid was extracted directly after injection of $1 \mathrm{ml}$ PBS with proteinase inhibitor. For each membrane, $500 \mu \mathrm{l}$ of protein solution was mixed with $500 \mu \mathrm{l}$ of sample buffer (array buffer 4 ) and $500 \mu \mathrm{l}$ of block buffer (array buffer 6). Fifteen mocroliters of reconstituted Mouse Cytokine Array Panel A Detection Antibody Cocktail was then added and incubated at room temperature for $1 \mathrm{~h}$. The array membrane was incubated with block buffer (array buffer 6) for $1 \mathrm{~h}$ on a rocking platform shaker. The tray should be oriented to ensure that each membrane rocks end to end in its well. The block buffer was then aspirated, and the prepared sample/antibody mixture was added onto the membrane and incubated overnight at room temperature on a rocking platform shaker. Each membrane was washed three times with $20 \mathrm{ml}$ of $1 \mathrm{X}$ wash buffer for $10 \mathrm{~min}$ on a rocking platform shaker. Membranes were incubated in $1.5 \mathrm{ml}$ of streptavidin-HRP (1:1,000 dilution) in array buffer 5 for $30 \mathrm{~min}$ at room temperature on a rocking platform shaker, and then washed with wash buffer three more times. Antibodyantigen complexes were visualized and analyzed using LI-COR C-Digit.

Histological analysis and immunostaining. The retrieval microcapsules were fixed in $4 \%$ paraformaldehyde, stacked together into Histogel, embedded in paraffin and then sectioned by Cornell Histology Core Facility. The samples were sliced on a microtome at a thickness of $5 \mu \mathrm{m}$. Paraffin sections were then stained with hematoxylin/eosin.

To conduct immunofluorescence staining of retrieved islets, the histological slides were deparaffinized by sequential washing in xylene, 100,90 , and $75 \%$ ethanol, and DI water. These slides were then boiled in $1 \mathrm{mM}$ EDTA for antigen exposure. Non-specific binding was blocked with $10 \%$ goat serum for $45 \mathrm{~min}$ at room temperature. After blocking, slides were decanted and incubated with primary rabbit anti-insulin antibodies $(1: 200)$ overnight at $4{ }^{\circ} \mathrm{C}$. The sections were then washed and incubated with the FITC-conjugated secondary antibodies, 594conjuaged donkey anti-rabbit igG (1:400 dilution) for $30 \mathrm{~min}$ at room temperature. Slides were washed twice with water, labeled with DAPI, and covered with coverslips. Fluorescence images were captured using an EVOS AMF4300 imaging system.

To conduct immunofluorescence staining of retrieved alginate microcapsules, samples were fixed in $4 \%$ paraformaldehyde overnight before use. Samples were washed with Krebs Buffer three times. Samples were then washed with PBS three times and a 1\% Triton X-100 solution was used to permeabilize cells. Samples were incubated for $15 \mathrm{~min}$ at room temperature. Samples were then transferred into $1 \%$ albumin solution for $30 \mathrm{~min}$ at room temperature. Next, the microcapsules were incubated for $1 \mathrm{~h}$ in an immunostaining cocktail solution consisting two drops of DAPI and specific antibodies (1:200) in 1\% albumin solution. Staining solution was then removed and washed twice with PBS solution containing $0.1 \%$ tween 20 . Samples were washed twice with PBS and then transferred into a concave glass slide. Excess PBS was removed, and 50\% glycerol solution was added. The samples were finally covered with coverslips. Fluorescence Images were captured under a Zeiss LSM710 confocal microscope at Cornell Biotechnology Resource Center Imaging Facility.

Evaluation of PCO. The retrieved microcapsules were evaluated using phasecontrast microscopy to determine the degree of $\mathrm{CO}$ following the previous method $^{43}$. For retrieved microcapsules, the degree of $\mathrm{CO}$ was determined by categorizing coverage percentage of surface area that was covered by PCO: $0-25$, $25-50,50-75,75-100 \%$. The PCO degree for retrieved capsules was calculated using the equation: PCO degree $(\%)=$ (the number of capsules assessed in each category/the total number of retrieved capsules) $\times 100$.

Statistical analysis. Data are expressed as Mean \pm SEM in these experiments. Paired Student's $t$-test was used to compare two small sets of quantitative data from surface roughness, mechanical property, TLRs studies, protein adsorption, macrophage activation studies, and ex vivo GSIS experiments, with $P<0.05$ being considered as statistically significant. For PCO quantification, Chi-squared test with Bonferroni correction was used to compare modified alginates to SLG20 alginate and calculate $P$ values. Differences between groups were considered statistically significant when $P<0.05$.

Reporting summary. Further information on research design is available in the Nature Research Reporting Summary linked to this article.
In vitro GSIS assay. Krebs Ringer Bicarbonate (KRB) buffer [98.5 mM NaCl $4.9 \mathrm{mM} \mathrm{KCl}, 2.6 \mathrm{mM} \mathrm{CaCl}_{2}, 1.2 \mathrm{mM} \mathrm{MgSO}_{4} \cdot 7 \mathrm{H}_{2} \mathrm{O}, 1.2 \mathrm{mM} \mathrm{KH}_{2} \mathrm{PO}_{4}$ and $25.9 \mathrm{mM}$ 


\section{Data availability}

All relevant data are available within the article and Supplementary Information, and from the corresponding author upon reasonable request.

Received: 17 March 2019; Accepted: 30 October 2019;

Published online: 20 November 2019

\section{References}

1. Atkinson, M. A., Eisenbarth, G. S. \& Michels, A. W. Type 1 diabetes. Lancet 383, 69-82 (2014).

2. Katsarou, A. et al. Type 1 diabetes mellitus. Nat. Rev. Dis. Prim. 3, 17016 (2017).

3. Desai, T. \& Shea, L. D. Advances in islet encapsulation technologies. Nat. Rev. Drug. Discov. 16, 338 (2017).

4. Orive, G. et al. Cell encapsulation: promise and progress. Nat. Med. 9, 104 (2003).

5. Scharp, D. W. \& Marchetti, P. Encapsulated islets for diabetes therapy: history, current progress, and critical issues requiring solution. Adv. Drug Deliv. Rev. 67, 35-73 (2014).

6. Weaver, J. D. et al. Vasculogenic hydrogel enhances islet survival, engraftment, and function in leading extrahepatic sites. Sci. Adv. 3, e1700184 (2017).

7. Vlahos, A. E., Cober, N. \& Sefton, M. V. Modular tissue engineering for the vascularization of subcutaneously transplanted pancreatic islets. Proc. Natl Acad. Sci. USA 114, 9337-9342 (2017).

8. Pedraza, E., Coronel, M. M., Fraker, C. A., Ricordi, C. \& Stabler, C. L. Preventing hypoxia-induced cell death in beta cells and islets via hydrolytically activated, oxygen-generating biomaterials. Proc. Natl Acad. Sci. USA 109, 4245-4250 (2012).

9. $\mathrm{Yu}, \mathrm{J}$. et al. Microneedle-array patches loaded with hypoxia-sensitive vesicles provide fast glucose-responsive insulin delivery. Proc. Natl Acad. Sci. USA 112, 8260-8265 (2015).

10. Veiseh, O., Tang, B. C., Whitehead, K. A., Anderson, D. G. \& Langer, R. Managing diabetes with nanomedicine: challenges and opportunities. Nat Rev. Drug. Discov. 14, 45 (2015).

11. Shapiro, A. J., Pokrywczynska, M. \& Ricordi, C. Clinical pancreatic islet transplantation. Nat. Rev. Endocrinol. 13, 268 (2017).

12. Pagliuca, F. W. et al. Generation of functional human pancreatic $\beta$ cells in vitro. Cell 159, 428-439 (2014).

13. Ma, H., Wert, K. J., Shvartsman, D., Melton, D. A. \& Jaenisch, R Establishment of human pluripotent stem cell-derived pancreatic $\beta$-like cells in the mouse pancreas. Proc. Natl Acad. Sci. USA 115, 3924-3929 (2018).

14. Orive, G. et al. Cell encapsulation: technical and clinical advances. Trends Pharmacol. Sci. 36, 537-546 (2015).

15. An, D. et al. Designing a retrievable and scalable cell encapsulation device for potential treatment of type 1 diabetes. Proc. Natl Acad. Sci. USA 115, E263-E272 (2018).

16. Lee, K. Y. \& Mooney, D. J. Alginate: properties and biomedical applications. Prog. Polym. Sci. 37, 106-126 (2012).

17. Utech, S. et al. Microfluidic generation of monodisperse, structurally homogeneous alginate microgels for cell encapsulation and 3D cell culture. Adv. Healthc. Mater. 4, 1628-1633 (2015).

18. Wang, H., Zhao, X. \& He, C. Constructing a novel zwitterionic surface of PVDF membrane through the assembled chitosan and sodium alginate. Int. J. Biol. Macromol. 87, 443-448 (2016).

19. Vegas, A. J. et al. Long-term glycemic control using polymer-encapsulated human stem cell-derived beta cells in immune-competent mice. Nat. Med. 22, 306 (2016).

20. Schneider, S. et al. Long-term graft function of adult rat and human islets encapsulated in novel alginate-based microcapsules after transplantation in immunocompetent diabetic mice. Diabetes 54, 687-693 (2005).

21. Sheikh, Z., Brooks, P. J., Barzilay, O., Fine, N. \& Glogauer, M. Macrophages, foreign body giant cells and their response to implantable biomaterials. Materials 8, 5671-5701 (2015).

22. Zhang, Y. et al. High-water-content and resilient PEG-containing hydrogels with low fibrotic response. Acta Biomater. 53, 100-108 (2017).

23. Vegas, A. J. et al. Combinatorial hydrogel library enables identification of materials that mitigate the foreign body response in primates. Nat. Biotechnol. 34, 345 (2016).

24. Bochenek, M. A. et al. Alginate encapsulation as long-term immune protection of allogeneic pancreatic islet cells transplanted into the omental bursa of macaques. Nat. Biomed. Eng. 2, 810 (2018).

25. Grainger, D. W. All charged up about implanted biomaterials. Nat. Biotechnol. 31, 507 (2013).

26. Zhang, L. et al. Zwitterionic hydrogels implanted in mice resist the foreignbody reaction. Nat. Biotechnol. 31, 553 (2013).
27. Swartzlander, M. D. et al. Linking the foreign body response and protein adsorption to PEG-based hydrogels using proteomics. Biomaterials 41, 26-36 (2015).

28. Jiang, S. \& Cao, Z. Ultralow-fouling, functionalizable, and hydrolyzable zwitterionic materials and their derivatives for biological applications. $A d v$ Mater. 22, 920-932 (2010).

29. Ladd, J., Zhang, Z., Chen, S., Hower, J. C. \& Jiang, S. Zwitterionic polymers exhibiting high resistance to nonspecific protein adsorption from human serum and plasma. Biomacromolecules 9, 1357-1361 (2008).

30. Goda, T., Konno, T., Takai, M., Moro, T. \& Ishihara, K. Biomimetic phosphorylcholine polymer grafting from polydimethylsiloxane surface using photo-induced polymerization. Biomaterials 27, 5151-5160 (2006).

31. Chien, H.-W., Tsai, W.-B. \& Jiang, S. Direct cell encapsulation in biodegradable and functionalizable carboxybetaine hydrogels. Biomaterials 33, 5706-5712 (2012)

32. Bai, T. et al. Restraint of the differentiation of mesenchymal stem cells by a nonfouling zwitterionic hydrogel. Angew. Chem. 126, 12943-12948 (2014).

33. Zhang, W., Yang, Z., Kaufman, Y. \& Bernstein, R. Surface and anti-fouling properties of a polyampholyte hydrogel grafted onto a polyethersulfone membrane. J. Colloid Interface Sci. 517, 155-165 (2018).

34. Shao, Q. \& Jiang, S. Molecular understanding and design of zwitterionic materials. Adv. Mater. 27, 15-26 (2015).

35. Smith, R. S. et al. Vascular catheters with a nonleaching poly-sulfobetaine surface modification reduce thrombus formation and microbial attachment. Sci. Transl. Med. 4, 153ra132-153ra132 (2012).

36. Lalani, R. \& Liu, L. Electrospun zwitterionic poly (sulfobetaine methacrylate) for nonadherent, superabsorbent, and antimicrobial wound dressing applications. Biomacromolecules 13, 1853-1863 (2012).

37. Tsai, W. B., Grunkemeier, J. M., McFarland, C. D. \& Horbett, T. A. Platelet adhesion to polystyrene-based surfaces preadsorbed with plasmas selectively depleted in fibrinogen, fibronectin, vitronectin, or von Willebrand's factor. $J$. Biomed. Mater. Res. 60, 348-359 (2002).

38. Luu, T. U., Gott, S. C., Woo, B. W., Rao, M. P. \& Liu, W. F. Micro-and nanopatterned topographical cues for regulating macrophage cell shape and phenotype. ACS Appl. Mater. interfaces 7, 28665-28672 (2015).

39. Paredes-Juarez, G., de Haan, B., Faas, M. \& de Vos, P. A technology platform to test the efficacy of purification of alginate. Materials 7, 2087-2103 (2014).

40. Kolb, M. et al. Differences in the fibrogenic response after transfer of active transforming growth factor- $\beta 1$ gene to lungs of "fibrosis-prone" and "fibrosisresistant" mouse strains. Am. J. Respir. Cell Mol. Biol. 27, 141-150 (2002).

41. Veiseh, O. et al. Size-and shape-dependent foreign body immune response to materials implanted in rodents and non-human primates. Nat. Mater. 14, 643 (2015).

42. Tuch, B. E. et al. Safety and viability of microencapsulated human islets transplanted into diabetic humans. Diabetes Care 32, 1887-1889 (2009).

43. Vaithilingam, V. et al. Beneficial effects of coating alginate microcapsules with macromolecular heparin conjugates-in vitro and in vivo study. Tissue Eng. Part. A. 20, 324-334 (2013).

44. Saurer, L. et al. TREM-1 promotes intestinal tumorigenesis. Sci. Rep. 7, 14870 (2017).

45. Hoshino, A. et al. Inhibition of CCL1-CCR8 interaction prevents aggregation of macrophages and development of peritoneal adhesions. J. Immunol. 178, 5296-5304 (2007)

46. Thiele, N. D. et al. TIMP-1 is upregulated, but not essential in hepatic fibrogenesis and carcinogenesis in mice. Sci. Rep. 7, 714 (2017).

47. Sawant, K. V. et al. Chemokine CXCL1 mediated neutrophil recruitment: Role of glycosaminoglycan interactions. Sci. Rep. 6, 33123 (2016).

48. Doloff, J. C. et al. Colony stimulating factor-1 receptor is a central component of the foreign body response to biomaterial implants in rodents and nonhuman primates. Nat. Mater. 16, 671 (2017).

49. Ivanov, $\mathrm{S}$. et al. Mesothelial cell CSF1 sustains peritoneal macrophage proliferation. Eur. J. Immunol. https://doi.org/10.1002/eji.201948164 (2019).

50. McFadden, C. et al. Preferential migration of T regulatory cells induced by IL16. J. Immunol. 179, 6439-6445 (2007).

51. Omer, A. et al. Long-term normoglycemia in rats receiving transplants with encapsulated islets. Transplantation 79, 52-58 (2005).

52. Krishnan, R. et al. in Cell Microencapsulation 305-333 (Springer, 2017).

53. Villa, C. et al. Effects of composition of alginate-polyethylene glycol microcapsules and transplant site on encapsulated islet graft outcomes in mice. Transplantation 101, 1025 (2017).

54. Toso, C. et al. Effect of microcapsule composition and short-term immunosuppression on intraportal biocompatibility. Cell Transplant. 14, 159-167 (2005)

55. Safley, S. A. et al. Microencapsulated adult porcine islets transplanted intraperitoneally in streptozotocin-diabetic non-human primates. Xenotransplantation 25, e12450 (2018). 
56. Duvivier-Kali, V. F., Omer, A., Parent, R. J., O’Neil, J. J. \& Weir, G. C. Complete protection of islets against allorejection and autoimmunity by a simple barium-alginate membrane. Diabetes 50, 1698-1705 (2001).

57. Sakai, S., Mu, C., Kawabata, K., Hashimoto, I. \& Kawakami, K. Biocompatibility of subsieve-size capsules versus conventional-size microcapsules. J. Biomed. Mater. Res. Part A. 78, 394-398 (2006).

58. Orive, G., Emerich, D. \& De Vos, P. Encapsulate this: the do's and don'ts. Nat. Med. 20, 233 (2014).

59. Dufrane, D., Goebbels, R.-M., Saliez, A., Guiot, Y. \& Gianello, P. Six-month survival of microencapsulated pig islets and alginate biocompatibility in primates: proof of concept. Transplantation 81, 1345-1353 (2006).

60. Köllmer, M., Appel, A. A., Somo, S. I. \& Brey, E. M. Long-term function of alginate-encapsulated islets. Tissue Eng. Part B: Rev. 22, 34-46 (2015).

61. Hall, K. K., Gattás-Asfura, K. M. \& Stabler, C. L. Microencapsulation of islets within alginate/poly (ethylene glycol) gels cross-linked via Staudinger ligation. Acta Biomater. 7, 614-624 (2011).

62. Krishnan, R. et al. Noninvasive evaluation of the vascular response to transplantation of alginate encapsulated islets using the dorsal skin-fold model. Biomaterials 35, 891-898 (2014).

63. Tam, S. et al. Biocompatibility and physicochemical characteristics of alginate-polycation microcapsules. Acta Biomater. 7, 1683-1692 (2011).

64. Safley, S. A., Cui, H., Cauffiel, S., Tucker-Burden, C. \& Weber, C. J. Biocompatibility and immune acceptance of adult porcine islets transplanted intraperitoneally in diabetic NOD mice in calcium alginate poly-L-lysine microcapsules versus barium alginate microcapsules without poly-L-lysine. J. Diabetes Sci. Technol. 2, 760-767 (2008).

65. Mørch, Ý. A., Donati, I., Strand, B. L. \& Skjåk-Bræk, G. Effect of Ca2+, Ba2+, and $\mathrm{Sr} 2+$ on alginate microbeads. Biomacromolecules 7, 1471-1480 (2006).

66. Park, H. S. et al. Antifibrotic effect of rapamycin containing polyethylene glycol-coated alginate microcapsule in islet xenotransplantation. J. Tissue Eng. Regen. Med. 11, 1274-1284 (2017).

67. Bünger, C. et al. Deletion of the tissue response against alginate-pll capsules by temporary release of co-encapsulated steroids. Biomaterials 26, 2353-2360 (2005).

68. Dufrane, D. et al. The influence of implantation site on the biocompatibility and survival of alginate encapsulated pig islets in rats. Biomaterials 27, 3201-3208 (2006).

69. Wang, T. et al. An encapsulation system for the immunoisolation of pancreatic islets. Nat. Biotechnol. 15, 358 (1997).

70. Jacobs-Tulleneers-Thevissen, D. et al. Sustained function of alginateencapsulated human islet cell implants in the peritoneal cavity of mice leading to a pilot study in a type 1 diabetic patient. Diabetologia 56, 1605-1614 (2013).

71. Wu, J., Lin, W., Wang, Z., Chen, S. \& Chang, Y. Investigation of the hydration of nonfouling material poly (sulfobetaine methacrylate) by low-field nuclear magnetic resonance. Langmuir 28, 7436-7441 (2012).

72. Díaz Blanco, C. et al. Building an antifouling zwitterionic coating on urinary catheters using an enzymatically triggered bottom-up approach. ACS Appl. Mater. interfaces 6, 11385-11393 (2014).

73. $\mathrm{Wu}, \mathrm{H}$. et al. Highly sensitive and stable zwitterionic poly (sulfobetaine-3, 4ethylenedioxythiophene)(PSBEDOT) glucose biosensor. Chem. Sci. 9, 2540-2546 (2018).

74. Lu, Y. et al. Micelles with ultralow critical micelle concentration as carriers for drug delivery. Nat. Biomed. Eng. 2, 318 (2018).

75. Muro, E. et al. Small and stable sulfobetaine zwitterionic quantum dots for functional live-cell imaging. J. Am. Chem. Soc. 132, 4556-4557 (2010).

76. Moro, T. et al. Surface grafting of artificial joints with a biocompatible polymer for preventing periprosthetic osteolysis. Nat. Mater. 3, 829 (2004).

77. Zhang, P. et al. Zwitterionic gel encapsulation promotes protein stability, enhances pharmacokinetics, and reduces immunogenicity. Proc. Natl. Acad. Sci. 112, 12046-12051 (2015).

78. Xue, Y. et al. A green and facile method for the preparation of a $\mathrm{pH}-$ responsive alginate nanogel for subcellular delivery of doxorubicin. RSC $A d v$. 5, 73416-73423 (2015)
79. Sahasrabudhe, N. M. et al. Dietary fiber pectin directly blocks Toll-like receptor 2-1 and prevents doxorubicin-induced ileitis. Front. Immunol. 9, 383 (2018).

80. Morgado, P., Ong, Y.-C., Boothroyd, J. C. \& Lodoen, M. B. Toxoplasma gondii induces B7-2 expression through activation of JNK signal transduction. Infect. Immun. 79, 4401-4412 (2011).

81. Lacy, P. E. \& Kostianovsky, M. Method for the isolation of intact islets of Langerhans from the rat pancreas. Diabetes 16, 35-39 (1967).

82. Ricordi, C. et al. Islet isolation assessment in man and large animals. Acta diabetologia Lat. 27, 185-195 (1990).

\section{Acknowledgements}

We would like to thank Drs. Cathrine Bundgaard's and Birgitte Viuff's assistance with surgery and necropsy for pig study. This work was partially provided by the Juvenile Diabetes Research Foundation (JDRF), the Hartwell Foundation, the National Institutes of Health (NIH, 1R01DK105967-01A1) and the Novo Nordisk Company.

\section{Author contributions}

Q.L. and M.M. conceived and designed the project. A.S., B.H., and P.V. performed capsule characterization. A.C. and D.A. performed in vivo experiments in mouse and analyzed the samples. L.W., W.S., M.Z., and X.W. performed immunohistochemical experiments and helped to draw schematic diagrams. J.F designed and performed the in vivo dog experiments. K.K., A.V., L.G., and C.R. performed the in vivo pig experiments and analyzed the samples. E.C. and W.L. performed macrophage activation studies. All authors reviewed the manuscript and provided input.

\section{Competing interests}

K.K., A.V., C.R., and L.G.G. are Novo Nordisk A/S employees and shareholders in the company. All other authors declare no competing interests.

\section{Additional information}

Supplementary information is available for this paper at https://doi.org/10.1038/s41467 019-13238-7.

Correspondence and requests for materials should be addressed to M.M.

Peer review information Nature Communications thanks Shaoyi Jiang and other, anonymous, reviewers for their contributions to the peer review of this work.

Reprints and permission information is available at http://www.nature.com/reprints

Publisher's note Springer Nature remains neutral with regard to jurisdictional claims in published maps and institutional affiliations.

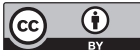

Open Access This article is licensed under a Creative Commons Attribution 4.0 International License, which permits use, sharing, adaptation, distribution and reproduction in any medium or format, as long as you give appropriate credit to the original author(s) and the source, provide a link to the Creative Commons license, and indicate if changes were made. The images or other third party material in this article are included in the article's Creative Commons license, unless indicated otherwise in a credit line to the material. If material is not included in the article's Creative Commons license and your intended use is not permitted by statutory regulation or exceeds the permitted use, you will need to obtain permission directly from the copyright holder. To view a copy of this license, visit http://creativecommons.org/ licenses/by/4.0/.

(C) The Author(s) 2019 